NORDITA-2001/11 HE

SPIN-2001/15

\title{
Supersymmetric 3-branes on smooth ALE manifolds with flux ${ }^{\dagger}$
}

\author{
M. Bertolini ${ }^{1}$, V. L. Campos ${ }^{2}$, G. Ferretti ${ }^{2}$, \\ P. Fré ${ }^{3}$, P. Salomonson ${ }^{2}$, M. Trigiante ${ }^{4}$ \\ 1 NORDITA, Blegdamsvej, 172100 Copenhagen Ø, Denmark \\ ${ }^{2}$ Institute for Theoretical Physics - Göteborg University and \\ Chalmers University of Technology, 41296 Göteborg, Sweden \\ ${ }^{3}$ Dipartimento di Fisica Teorica, Universitá di Torino, \& INFN - Sezione di Torino \\ via P. Giuria 1, I-10125 Torino, Italy \\ ${ }^{4}$ Spinoza Institute, Leuvenlaan 4, Utrecht, The Netherlands
}

\begin{abstract}
We construct a new family of classical BPS solutions of type IIB supergravity describing 3 -branes transverse to a 6 -dimensional space with topology $\mathbb{R}^{2} \times \mathrm{ALE}$. They are characterized by a non-trivial flux of the supergravity 2-forms through the homology 2-cycles of a generic smooth ALE manifold. Our solutions have two Killing spinors and thus preserve $\mathcal{N}=2$ supersymmetry. They are expressed in terms of a quasi harmonic function $H$ (the "warp factor"), whose properties we study in the case of the simplest ALE, namely the EguchiHanson manifold. The equation for $H$ is identified as an instance of the confluent Heun equation. We write explicit power series solutions and solve the recurrence relation for the coefficients, discussing also the relevant asymptotic expansions. While, as in all such $\mathcal{N}=2$ solutions, supergravity breaks down near the brane, the smoothing out of the vacuum geometry has the effect that the warp factor is regular in a region near the cycle. We interpret the behavior of the warp factor as describing a three-brane charge "smeared" over the cycle and consider the asymptotic form of the geometry in that region, showing that conformal invariance is broken even when the complex type IIB 3-form field strength is assumed to vanish. We conclude with a discussion of the basic features of the gauge theory dual.
\end{abstract}

PACS: $11.25 . \mathrm{Hf}$

Keywords: 3-brane, ALE, flux

${ }^{\dagger}$ This work is supported in part by the European Union RTN contracts HPRN-CT-2000-00122 and HPRN-CT2000-00131. 


\section{Contents}

\begin{tabular}{lll}
\hline 1 & Introduction & 1
\end{tabular}

\begin{tabular}{|lll}
2 & Bosonic action and field equations of type IIB supergravity & 3
\end{tabular}

\begin{tabular}{|lll}
\hline 3 & 3 -brane solution with ALE flux & 4
\end{tabular}

3.1 Solution of the bosonic field equations . . . . . . . . . . . . . . . . . . . . . . . . 5

3.2 Proof of bulk supersymmetry . . . . . . . . . . . . . . . . . . . . . . . . . 10

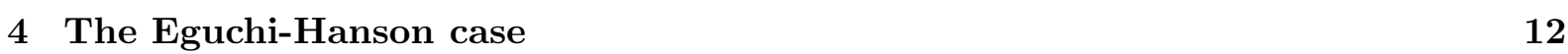

4.1 Solution method by partial Fourier transform . . . . . . . . . . . . . . . . 13

4.2 Asymptotics of the the two independent solutions $u_{1}, u_{2}$ for the homogeneous

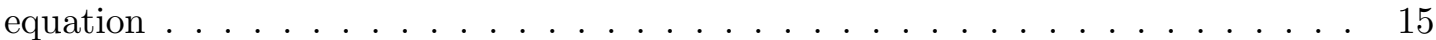

4.3 The 3-brane charge, the physical boundary conditions and the final form of the Green function . . . . . . . . . . . . . . . . . . . . . 17

4.4 Reduction to the confluent Heun equation . . . . . . . . . . . . . . . . . . . . . . 18

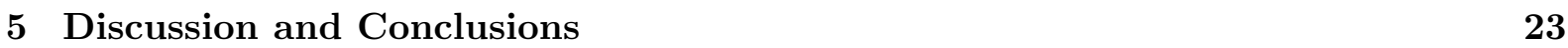

\begin{tabular}{|lr}
\hline A Notations and Conventions & 26
\end{tabular}

\begin{tabular}{|lr}
\hline B The Eguchi-Hanson space & 28
\end{tabular}

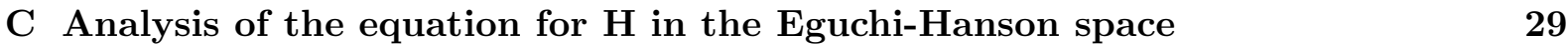

\section{Introduction}

After the seminal paper by Maldacena [1], many efforts have been devoted to extend the gauge/gravity correspondence to less supersymmetric and non-conformal cases. In this context considerable attention was recently directed to the study of fractional branes [2]-15]. These are the natural elementary D-branes occurring whenever string theory is reduced on a (not necessarily compact) orbifold [16], [17]- 20]. Because of their nature they cannot move away from the orbifold apex and thus the dual gauge theory on their world-volume lacks the relevant moduli fields. Generically, this leads to both reduced supersymmetry and non-vanishing $\beta$-functions. Most interesting are the fractional D3-branes, namely the case when the world-volume theory is four-dimensional. In this respect the two most appealing situations are provided by the $\mathcal{N}=1$ case emerging from singular limits of $\mathrm{CY}$ spaces and the $\mathcal{N}=2$ case arising from the singular limit of ALE spaces. Much work was devoted to both.

A common feature of many supergravity solutions representing non-conformal situations is the presence of naked singularities of repulson type [21]. These correspond to IR singularities at the gauge theory level and one expects that they should be resolved or explained by some stringy effect. Although a general recipe does not seem to exist, progress in understanding such an issue was made both for the $\mathcal{N}=1$ and the $\mathcal{N}=2$ case.

In constructing supergravity solutions corresponding to fractional branes one proceeds in two steps. Firstly, one defines the vacuum solution as a space-time obtained by quotiening ten-dimensional Minkowski space-time modulo a discrete group with all other fields set to their constant background values (typically, the latter are zero with the exception of the dilaton, which 
sets the string coupling, and the NS-NS 2-form $B$, which determines whether the conformal field theory is well defined or not [22]). Secondly, one places the branes at the orbifold/conifold apex, which has the effect of turning on the relevant gauge fields and of warping the metric in a way that makes the curvature non-zero even away from the singularity. As already stressed, these solutions generally display singularities (see [3] for the $\mathcal{N}=1$ case and [7] for the $\mathcal{N}=2$ case).

Once confronted with superstring theory on such a space-time, it is a natural question to ask whether this space can be thought of as a singular limit of a smooth geometry. This can always be achieved when no branes are present. If the smoothing out can be carried out even in the presence of branes, and if the warp factor is such that the curvature remains small everywhere and the singularities in the solution are removed, then one can claim that the supergravity solution provides a dual description of the gauge theory. This is what was accomplished, for instance, in the $\mathcal{N}=1$ deformed conifold solution of Klebanov and Strassler [5], where the dynamics of the brane deforms the conifold at its apex, yielding a singularity free solution.

What is somewhat frustrating is that, contrary to what one would naively expect, the $\mathcal{N}=1$ case turned out to be more amenable to a smooth supergravity analysis than the $\mathcal{N}=2$ case. Actually, for the $\mathcal{N}=2$ case, a completely different phenomenon has been shown to occur [7]-[11]: the singular region is excised by the enhançon mechanism [23], which corresponds to a locus where extra massless degrees of freedom come into play, thus completely changing the geometry in the interior. From the gauge theory point of view the enhançon is the scale at which the gauge coupling diverges and where instantonic effects become relevant.

Despite this specific feature of the $\mathcal{N}=2$ case, one could anyhow try to see if better behaved solutions can arise when considering, analogously to what has been done in the conifold case in [5] and [6], the blow-up of the singular space, namely the smooth ALE manifold. Then one could try to see if there is any relation between the enhançon and the geometric moduli of the better behaved geometry, try to disentangle the singular properties of the solution, and eventually investigate their meaning in the context of the dual gauge theory.

Starting from the singular space $\mathbb{C}^{2} / \Gamma$, where $\Gamma$ is a discrete subgroup of $S U(2)$, one should inquire whether one can soften the vacuum geometry to a smooth ALE space. It is well known that this cannot be done in a supersymmetric way if fractional D3-branes are present and if the string theory is really at the orbifold point, that is, with a non zero background $B$-field threaded through the vanishing cycle of the orbifold. There are many arguments for such a "no-go" theorem. Probably the most intuitive one involves going to the T-dual type IIA picture, 24]. There one finds two NS5-branes with a stack of D4-branes (dual to the fractional D3-branes) stretched between them. The blowing up of the orbifold corresponds to moving the NS5-branes in the direction transverse to the D4-branes, and this involves stretching the D4-branes even further, thus increasing the energy of the system and hence breaking supersymmetryt.

Yet the above argument does not rule out the existence of supersymmetric bulk solutions of type IIB supergravity that smooth out the quotient. Such solutions, which eventually have to be interpreted as suitable bound states of some D-brane state, are precisely those that we find in the present paper.

In particular, we find $\mathcal{N}=2$ BPS solutions corresponding to 3-brane states where the transverse space is given by $\mathbb{R}^{2} \times \mathrm{ALE}$ and where there is a non zero value of the complex type IIB supergravity 3 -form in the transverse directions (note that this is the distinctive feature of fractional D-branes in the singular orbifold theories). As already noticed, solutions of this type with $\mathcal{N}=1$ supersymmetry have appeared in the recent literature but they were mostly

\footnotetext{
${ }^{1}$ We thank R. Russo for a discussion on this point.
} 
concerned with the case where the six-dimensional transverse space was a cone. This had the simplifying feature of making the warp factor depend only on one (radial) coordinate. This feature cannot be preserved if one imposes $\mathcal{N}=2$ supersymmetry since the required $\mathrm{SU}(2)$ holonomy forces the geometry of the transverse space to be of the factorized form described above. The price to pay is that the warp factor is now dependent on (at least) two variables and it is governed by a complicated partial differential equation. In fact the whole set of IIB equations can be reduced to the solution of such an equation for the warp factor, whose source is essentially dictated by supersymmetry up to an arbitrary analytic function $\gamma(z)$.

Supergravity alone is not sufficient to determine $\gamma(z)$ or the boundary conditions. This arbitrariness implies that our solution describes various deformation or various vacua of $\mathcal{N}=2$ theories. The case $\gamma(z)=$ const. describes a vanishing three-form flux and corresponds to the well known conformal $\mathcal{N}=2$ theory with product gauge group $\mathrm{U}(\mathrm{N}) \times \mathrm{U}(\mathrm{N})$, hyper-multiplets in the bi-fundamental representation and Fayet-Iliopoulos terms describing the ALE moduli. The new ingredient in our construction is the following. We show how at the supergravity level a three-form flux can be turned on consistently with supersymmetry. Some consequences of this for the gauge dual theory are analyzed in the concluding section yet a complete analysis of such an issue involves a systematic study of possible supersymmetric world-volume actions of branes on arbitrary curved backgrounds (in particular ALE manifolds) which is work in progress and the subject of a forthcoming publication.

The paper is organized as follows. In section 2 we briefly recall the structure of the equations of motion of type IIB supergravity, fixing our conventions for the fields. In section 3, after studying the general set-up for arbitrary ALE spaces, we give the structure of our 3-brane solution whose distinctive feature is the localized 3-form flux along any exceptional compact 2-cycle of the ALE space. Finally, applying a criterion which is analogous to that discussed in [25]-28], (see also [29]) we give the proof of its supersymmetry (in this respect, our solutions fall in the so called type B class [30], characterized by the 3-form to be imaginary self-dual). In order to consider an explicit example, in section 1 we specialize to the simplest such space, the Eguchi-Hanson space, and study in detail the properties of the warp factor. Finally, in section 5 we summarize our results, discuss the relevant physical properties of our solutions, discuss some basic aspects of their gauge theory dual and conclude with some comments on open issues.

\section{Bosonic action and field equations of type IIB supergravity}

As it is well known, type IIB supergravity does not have any conventional supersymmetric action. However, as it happens for all on-shell supergravity theories, the complete set of field equations can be obtained as consistency conditions from the closure of the supersymmetry transformation algebra [31]. In the case of type IIB supergravity, one was also able [32] to obtain a complete, manifestly $\mathrm{SU}(1,1)$-covariant formulation of the theory based on the rheonomic approach to supergravity theory [33].

The bosonic part of the equations can be formally obtained through variation of the following action 2:

$S_{\text {IIB }}=\frac{1}{2 \kappa^{2}}\left\{\int d^{10} x[-2 \sqrt{-\operatorname{det} g} R]-\frac{1}{2} \int\left[d \varphi \wedge \star d \varphi+\mathrm{e}^{-\varphi} F_{[3]}^{N S} \wedge \star F_{[3]}^{N S}+\mathrm{e}^{2 \varphi} F_{[1]}^{R R} \wedge \star F_{[1]}^{R R}\right.\right.$

\footnotetext{
${ }^{2}$ Note that our $R$ is equal to $-\frac{1}{2} R^{\text {old }}, R^{\text {old }}$ being the normalization of the scalar curvature usually adopted in General Relativity textbooks. The difference arises because in the traditional literature the Riemann tensor is not defined as the components of the curvature 2 -form $R^{a b}$ rather as -2 times such components.
} 


$$
\left.\left.+\mathrm{e}^{\varphi} F_{[3]}^{R R} \wedge \star F_{[3]}^{R R}+\frac{1}{2} F_{[5]}^{R R} \wedge \star F_{[5]}^{R R}-C_{[4]} \wedge F_{[3]}^{N S} \wedge F_{[3]}^{R R}\right]\right\}
$$

where:

$$
\begin{aligned}
& F_{[1]}^{R R}=d C_{[0]} \\
& F_{[3]}^{N S}=d B_{[2]} \\
& F_{[3]}^{R R}=d C_{[2]}-C_{[0]} d B_{[2]} \\
& F_{[5]}^{R R}=d C_{[4]}-\frac{1}{2}\left(B_{[2]} \wedge d C_{[2]}-C_{[2]} \wedge d B_{[2]}\right)
\end{aligned}
$$

It is important to stress though that the action (2.1) is to be considered only a book keeping device since the 4 -form $C_{[4]}$ is not free, its field strength $F_{[5]}^{R R}$ being subject to the on-shell self-duality constraint:

$$
F_{[5]}^{R R}=\star F_{[5]}^{R R}
$$

¿From the above action the corresponding equations of motion can be obtained:

$$
\begin{aligned}
& d \star d \varphi-e^{2 \varphi} F_{[1]}^{R R} \wedge \star F_{[1]}^{R R}=-\frac{1}{2}\left(e^{-\varphi} F_{[3]}^{N S} \wedge \star F_{[3]}^{N S}-e^{\varphi} F_{[3]}^{R R} \wedge \star F_{[3]}^{R R}\right) \\
& d\left(e^{2 \varphi} \star F_{[1]}^{R R}\right)=-e^{\varphi} F_{[3]}^{N S} \wedge \star F_{[3]}^{R R} \\
& d\left(e^{-\varphi} \star F_{[3]}^{N S}\right)+e^{\varphi} F_{[1]}^{R R} \wedge \star F_{[3]}^{R R}=-F_{[3]}^{R R} \wedge F_{[5]}^{R R} \\
& d\left(e^{\varphi} \star F_{[3]}^{R R}\right)=-F_{[5]}^{R R} \wedge F_{[3]}^{N S} \\
& d \star F_{[5]}^{R R}=-F_{[3]}^{N S} \wedge F_{[3]}^{R R} \\
& -2 R_{M N}=\frac{1}{2} \partial_{M} \varphi \partial_{N} \varphi+\frac{e^{2 \varphi}}{2} \partial_{M} C_{[0]} \partial_{N} C_{[0]}+150 F_{[5] M \ldots . . .} F_{[5] N} \ldots \\
& +9\left(e^{-\varphi} F_{[3] M . .}^{N S} F_{[3] N}^{N S} \cdot \cdot+e^{\varphi} F_{[3] M . .}^{R R} F_{[3] N}^{R R} \quad \cdot\right) \\
& -\frac{3}{4} g_{M N}\left(e^{-\varphi} F_{[3] \cdots}^{N S} F_{[3]}^{N S \cdots}+e^{\varphi} F_{[3] \cdots}^{R R} F_{[3]}^{R R \cdots}\right)
\end{aligned}
$$

It is not difficult to show, upon suitable identification of the massless superstring fields, that this is the correct set of equations which can be consistently obtained from the manifestly $\mathrm{SU}(1,1)$ covariant formulation of type IIB supergravity [32].

\section{3-brane solution with ALE flux}

In this section we provide the BPS solution corresponding to a 3-brane transverse to a smooth ALE space, namely we construct type IIB supergravity solutions describing 3 branes on a vacuum $\mathbb{R}^{1,3} \times \mathbb{R}^{2} \times$ ALE. This will be achieved without an analysis of the specific form of the worldvolume action of the brane, i.e. of the source term. Our physical assumption will just be that, together with the usual RR 5-form flux, the 3-brane solution has a non-trivial flux of the supergravity 2 -form potentials along (one of) the compact two cycle(s) of the blown-up orbifold (this translates into a non-vanishing value of the complex 3 -form field strength). 


\subsection{Solution of the bosonic field equations}

We separate the ten coordinates of space-time into the following subsets:

$$
x^{M}=\left\{\begin{array}{cl}
x^{\mu} \quad \mu=0,1,2,3 & \text { coordinates of the 3-brane world volume } \\
z=x^{4}+\mathrm{i} x^{5} & \text { complex coordinate of } \mathbb{R}^{2} \sim \mathbb{C} \\
y^{\tau} \quad \tau=6,7,8,9 & \text { real coordinates of the ALE 4-space }
\end{array}\right.
$$

and we make the following ansatz for the metric;:

$$
\begin{aligned}
d s^{2} & =H^{-\frac{1}{2}}\left(-\eta_{\mu \nu} d x^{\mu} d x^{\nu}\right)+H^{\frac{1}{2}} d z d \bar{z}+H^{\frac{1}{2}} d s_{A L E}^{2} \\
H & =H(z, \bar{z}, y) \\
d s_{A L E}^{2} & =g_{\tau \sigma}^{A L E} d y^{\tau} d y^{\sigma} \\
\operatorname{det}(g) & =H \operatorname{det}\left(g^{A L E}\right) \\
\eta_{\mu \nu} & =\operatorname{diag}(+,-,-,-)
\end{aligned}
$$

where $g_{\tau \sigma}^{A L E}$ is the metric of any ALE space and we denote the six-manifold spanned by $z, \bar{z}$ and $y^{\tau}$ by

$$
M_{6}=\mathbb{R}^{2} \times \mathrm{ALE}
$$

In terms of vielbein the ansatz (3.2) corresponds to

$$
V^{A}=\left\{\begin{array}{l}
V^{a}=H^{-1 / 4}(z, \bar{z}, y) d x^{a} \\
V^{i}=H^{1 / 4}(z, \bar{z}, y) e^{i}
\end{array}\right.
$$

where $e^{i}$ are the vielbein 1-forms of the manifold $M_{6}$. The structure equations of the latter aref:

$$
\begin{aligned}
0 & =d e^{i}-\widehat{\omega}^{i j} \wedge e^{k} \eta_{j k} \\
\widehat{R}^{i j} & =d \widehat{\omega}^{i j}-\widehat{\omega}^{i k} \wedge \widehat{\omega}^{\ell j} \eta_{k \ell}=\widehat{R}_{\ell m}^{i j} e^{\ell} \wedge e^{m}
\end{aligned}
$$

The relevant property of the $M_{6}$ metric that we use in solving the Einstein's equations is that it is Ricci flat:

$$
\widehat{R}_{\ell m}^{i m}=0
$$

What we need in order to derive our solution and discuss its supersymmetry properties is the explicit form of the spin connection for the full 10-dimensional metric (3.2) and the corresponding Ricci tensor. From the torsion equation one can uniquely determine the solution:

$$
\begin{aligned}
\omega^{a b} & =0 \\
\omega^{a i} & =\frac{1}{4} H^{-3 / 2} d x^{a} \eta^{i k} \partial_{k} H \\
\omega^{i j} & =\widehat{\omega}^{i j}+\Delta \omega^{i j} ; \quad \Delta \omega^{i j}=-\frac{1}{2} H^{-1} e^{[i} \eta^{j] k} \partial_{k} H
\end{aligned}
$$

\footnotetext{
${ }^{3}$ As explained in appendix A, the conventions for the gamma matrices and the spinors are set with a mostly minus metric $d \tau^{2}$. In the discussion of the solution, however, we use $d s^{2}=-d \tau^{2}$ for convenience. We hope this does not cause any confusion.

${ }^{4}$ The hats over the spin connection and the Riemann tensor denote quantities computed without the warp factor.
} 
Inserting this result into the definition of the curvature 2-form we obtain:

$$
\begin{aligned}
R_{b}^{a} & =-\frac{1}{8}\left[H^{-3 / 2} \square_{6} H-H^{-5 / 2} \partial_{k} H \partial^{k} H\right] \delta_{b}^{a} \\
R_{i}^{a} & =0 \\
R_{j}^{i} & =\frac{1}{8} H^{-3 / 2} \square_{6} H \delta_{j}^{i}-\frac{1}{8} H^{-5 / 2} \partial_{k} H \partial^{k} H \delta_{j}^{i}+\frac{1}{4} H^{-5 / 2} \partial_{j} H \partial^{i} H
\end{aligned}
$$

where

$$
\square_{6} \equiv \square_{\mathbb{R}^{2}}+\square_{\mathrm{ALE}}
$$

is the Laplacian on the tensor product space (3.4). The equations of motion for the scalar fields $\varphi$ and $C_{[0]}$ and for the 3 -form field strength $F_{[3]}^{N S}$ and $F_{[3]}^{R R}$ can be better analyzed introducing a complex notation. Defining

$$
\begin{aligned}
\mathcal{H}_{ \pm} & = \pm 2 e^{-\varphi / 2} F_{[3]}^{N S}+\mathrm{i} 2 e^{\varphi / 2} F_{[3]}^{R R} \\
P & =\frac{1}{2} d \varphi-\mathrm{i} \frac{1}{2} e^{\varphi} F_{[1]}^{R R}
\end{aligned}
$$

eq.s (2.4)-(2.7) can be written respectively as:

$$
\begin{array}{r}
d(\star P)-\mathrm{i} e^{\varphi} d C_{[0]} \wedge \star P+\frac{1}{16} \mathcal{H}_{+} \wedge \star \mathcal{H}_{+}=0 \\
d \star \mathcal{H}_{+}-\frac{\mathrm{i}}{2} e^{\varphi} d C_{[0]} \wedge \star \mathcal{H}_{+}=\mathrm{i} F_{[5]}^{R R} \wedge \mathcal{H}_{+}-P \wedge \star \mathcal{H}_{-}
\end{array}
$$

while eq. (2.8) for the 5-form becomes:

$$
d \star F_{[5]}^{R R}=\mathrm{i} \frac{1}{8} \mathcal{H}_{+} \wedge \mathcal{H}_{-}
$$

Besides assuming the structure (3.2) we also assume that the two scalar fields, namely the dilaton $\varphi$ and the Ramond-Ramond 0 -form $C_{[0]}$ are constant and vanishing:

$$
\varphi=0 \quad ; \quad C_{[0]}=0
$$

As we shall see, this assumption simplifies considerably the equations of motion.

The basic ansatz characterizing our solution and providing its interpretation as a 3-brane with a flux through a homology 2-cycle of the ALE space is given by the following:

$$
\begin{aligned}
& \mathcal{H}_{+}=2 d \gamma_{I}(z, \bar{z}) \wedge \omega^{I} \\
& \mathcal{H}_{-}=-2 d \bar{\gamma}_{I}(z, \bar{z}) \wedge \omega^{I}
\end{aligned}
$$

where $\gamma_{I}(z, \bar{z})$ is a complex field depending only on the coordinates $z, \bar{z}$ of $\mathbb{R}^{2}$, while $\omega^{I}$ ( $I=$ $1, \ldots, k)$ constitute a basis for the space of square integrable, anti-self-dual, harmonic forms on the ALE manifold. Notice that upon the ansatz (3.16), $\mathcal{H}_{+}$equals the usual complex 3 -form $G_{[3]}$ made up by the R-R and NS-NS type IIB 3 -forms. More precisely $\mathcal{H}_{+}=2 G_{[3]}$ while $\mathcal{H}_{-}=-2 \bar{G}_{[3]}$. From eq.(3.17) one then sees that our solutions fall in the same class of solutions discussed in [30], dubbed type B solutions, which are dual to M-theory solutions found by Becker and Becker in [34]. The former are in fact characterized by the 3 -form $G_{[3]}$ being a self-dual $(2,1)$ form. 
As it is well known 5 a smooth ALE manifold, arising from the resolution of a $\mathbb{C}^{2} / \Gamma$ singularity, where $\Gamma \subset \mathrm{SU}(2)$ is a discrete Kleinian group, has Hirzebruch signature:

$$
\tau=(\# \text { of conjugacy classes of } \Gamma)-1=\operatorname{rank} \text { of } \mathbb{G}(\Gamma)
$$

In the above formula $\mathbb{G}(\Gamma)$ is the simply laced Lie algebra corresponding to $\Gamma$ in the $\mathrm{ADE}$ classification of Kleinian groups, based on the Mac Kay correspondence [35. As a result of eq. 3.18) the ALE manifold that is HyperKähler admits a triplet of self-dual 2-forms that are non-integrable and exactly $\tau$ integrable anti-self-dual harmonic 2-forms. For these latter one can choose a basis $\omega^{I}$ that is dual to the integral homology basis of 2-cycles $\Sigma_{I}$ whose intersection matrix is the Cartan matrix $\mathcal{C}$ of $\mathbb{G}(\Gamma)$. Explicitly we can write:

$$
\begin{aligned}
\int_{\Sigma_{K}} \omega^{I} & =\delta_{K}^{I} \\
\int_{A L E} \omega^{I} \wedge \omega^{J} & =-\mathcal{C}^{-1 I J} \\
\omega^{I} \wedge \omega^{J} & =-\Delta^{I J}(y) \Omega_{A L E}
\end{aligned}
$$

where $\mathcal{C}^{I J}$ is the Cartan matrix of the corresponding (non-extended) ADE Dynkin diagram and $\Delta^{I J}(y)$ is a positive definite matrix whose entries are functions of the ALE space coordinates $y$ 's. The anti-self-duality of the $\omega^{I}$ guarantees that $\int \omega^{I} \wedge \star \omega^{J}$ is positive definite. Finally, following the conventions of appendix A (see in particular eq. (A.5) ) $\Omega_{A L E}$ is the volume 4-form of the ALE manifold.

If we insert the ansätze (3.16, 3.17) into the scalar field equations (2.4, 2.5) we obtain:

$$
\mathcal{H}_{+} \wedge \star \mathcal{H}_{+}=0
$$

which in turn implies that $\partial_{z} \gamma_{I} \bar{\partial}_{\bar{z}} \gamma_{J}=0$. This equation is solved by choosing $\gamma_{I}$ to be holomorphic:

$$
\bar{\partial} \gamma_{I}=0 \quad \text { where } \quad \partial=d z \frac{\partial}{\partial z} \quad ; \quad \bar{\partial}=d \bar{z} \frac{\partial}{\partial \bar{z}}
$$

Next we consider the self-dual 5 -form $F_{[5]}^{R R}$ which, because of its definition, must satisfy the following Bianchi identity:

$$
d F_{[5]}^{R R}=\mathrm{i} \frac{1}{8} \mathcal{H}_{+} \wedge \mathcal{H}_{-}
$$

Our ansatz for $F_{[5]}^{R R}$ is the following: (The volume forms $\Omega$ are described in appendix A)

$$
\begin{aligned}
F_{[5]}^{R R} & =\alpha(U+\star U) \\
U & =d\left(H^{-1} \Omega_{\mathbb{R}^{1,3}}\right)
\end{aligned}
$$

where $\alpha$ is a constant to be determined later. By construction $F_{[5]}^{R R}$ is self-dual and its equation of motion is trivially satisfied. What is not guaranteed is that also the Bianchi identity (3.22) is fulfilled. Imposing it, results into a differential equation for the function $H(z, \bar{z}, y)$. Using the Hodge duality relations (A.5) listed in appendix A we easily get:

$$
\star U=\mathrm{i} \partial H \wedge \Omega_{A L E}-\mathrm{i} \bar{\partial} H \wedge \Omega_{A L E}+\frac{\mathrm{i}}{2}\left(\hat{\star} d_{A L E} H\right) \wedge d z \wedge d \bar{z}
$$

\footnotetext{
${ }^{5}$ See for instance [16] for an early summary of ALE geometry in relation with superstrings and conformal field theories. This relation was developed in [17, 18] and is of primary relevance in connection with D-branes.
} 
Taking the exterior derivative of (3.24) we get:

$$
\begin{gathered}
d \star U=2 \mathrm{i} \bar{\partial} \partial H \wedge \Omega_{A L E}+\frac{\mathrm{i}}{2}\left(d_{(A L E)} \hat{\star} d_{(A L E)} H\right) \wedge d z \wedge d \bar{z}= \\
\left(\square_{\mathbb{R}^{2}} H+\square_{A L E} H\right) \frac{\mathrm{i}}{2} d \bar{z} \wedge d z \wedge \Omega_{A L E}
\end{gathered}
$$

Since $d F_{[5]}^{R R}=\alpha d \star U$ and

$$
\mathcal{H}_{+} \wedge \mathcal{H}_{-}=-4 \partial_{z} \gamma_{I} \partial_{\bar{z}} \bar{\gamma}_{J} \Delta^{I J} d \bar{z} \wedge d z \wedge \Omega_{A L E}
$$

we conclude that the Bianchi identity (3.22) is satisfied by our ansatz if:

$$
\left(\square_{\mathbb{R}^{2}}+\square_{A L E}\right) H=-\frac{1}{\alpha} \partial_{z} \gamma_{I} \partial_{\bar{z}} \bar{\gamma}_{J} \Delta^{I J}(y)
$$

This is the main differential equation the entire construction of our sought for 3-brane solution can be reduced to. We are going to show that the parameter $\alpha$ is determined by Einstein's equation and fixed to $\alpha=1$.

Let us consider next the field equation for the complex three-form, namely eq. (2.6) and (2.7). Since the two scalar fields are constant we have:

$$
d \star \mathcal{H}_{+}=\mathrm{i} F_{[5]}^{R R} \wedge \mathcal{H}_{+}
$$

Using our ansatz we immediately obtain:

$$
\begin{aligned}
d \star \mathcal{H}_{+} & =-2 \mathrm{i} H^{-2} d H \wedge \partial \gamma_{I} \wedge \omega^{I} \wedge \Omega_{\mathbb{R}^{1,3}}+2 \mathrm{i} H^{-1} \bar{\partial} \wedge \partial \gamma_{I} \wedge \Omega_{\mathbb{R}^{1,3}} \\
\mathrm{i} F_{[5]}^{R R} \wedge \mathcal{H}_{+} & =-2 \alpha \mathrm{i} H^{-2} d H \wedge \partial \gamma_{I} \wedge \omega^{I} \wedge \Omega_{\mathbb{R}^{1,3}}
\end{aligned}
$$

Hence if $\alpha=1$, as we have announced, the field equation for the fields $\gamma_{I}(z)$ reduces to:

$$
\bar{\partial} \wedge \partial \gamma_{I}=0 \Rightarrow \square_{\mathbb{R}^{2}} \gamma_{I}=0
$$

This equation has to be appropriately interpreted. It says that $\gamma_{I}$ are harmonic functions in twodimensions as the real and imaginary parts of any holomorphic function $\gamma_{I}(z)$ certainly are. The bulk equations do not impose any additional constraint besides this condition of holomorphicity. However, in presence of a boundary action for the 3 brane, the equation will be modified into:

$$
\square_{\mathbb{R}^{2}} \gamma_{I}=j_{I}(z)
$$

$j_{I}(z)$ being a source term, typically a delta function. In this case $\gamma_{I}$ is fixed as:

$$
\gamma_{I}(z)=\int G_{\mathbb{R}^{2}}\left(z, z^{\prime}\right) j_{I}\left(z^{\prime}\right) d z^{\prime}
$$

where $G_{\mathbb{R}^{2}}\left(z, z^{\prime}\right)$ is the Green function of the $\mathbb{R}^{2}$ Laplacian in complex coordinates and turns out to be proportional to $\log z$. From this point of view one could already expect the enhançon mechanism to be at work in a very similar way as in the orbifold case [7], [15]. We will come back to this issue in section 4 .

In order to show that also the Einstein's equation is satisfied by our ansatz we have to calculate the (trace subtracted) stress energy tensor of the five and three index field strengths. 
For this purpose we need the components of $F_{[5]}^{R R}$. These are easily dealt with. Relying on the ansatz (3.23) and on eq.(3.5) for the vielbein we immediately get:

$$
F_{A_{1} \ldots A_{5}}=\left\{\begin{array}{ccc}
F_{i a b c d} & = & \frac{1}{4 !} f_{i} \epsilon_{a b c d} \\
F_{j_{1} \ldots i_{5}} & = & \frac{1}{5 !} \epsilon_{i j_{1} \ldots j_{5}} f^{i} \\
\text { otherwise } & = & 0
\end{array}\right.
$$

where:

$$
f_{i}=-\alpha H^{-5 / 4} \partial_{i} H
$$

Then by straightforward algebra we obtain:

$$
\begin{aligned}
T_{b}^{a}\left[F_{[5]}^{R R}\right] & \equiv-75 F^{a \cdots} F_{b} \ldots=-\frac{1}{8} \delta_{b}^{a} f_{p} f^{p} \\
& =-\alpha^{2} \frac{1}{8} \delta_{b}^{a} H^{-5 / 2} \partial_{p} H \partial^{p} H \\
T_{j}^{i}\left[F_{[5]}^{R R}\right] & \equiv-75 F^{i \cdots \cdots} F_{j} \ldots=\frac{1}{4} f^{i} f_{j}-\frac{1}{8} \delta_{j}^{i} f_{p} f^{p} \\
& =\alpha^{2}\left[\frac{1}{4} H^{-5 / 4} \partial^{i} H \partial_{j} H-\frac{1}{8} \delta_{j}^{i} H^{-5 / 4} \partial^{p} H \partial_{p} H\right]
\end{aligned}
$$

Let us next consider the complex 3 -forms $\mathcal{H}_{ \pm}$. With our ansatz (3.17) they satisfy the 6dimensional self-duality relation on $M_{6}$ :

$$
\widehat{\star}_{6} \mathcal{H}_{ \pm}= \pm \mathrm{i} \mathcal{H}_{ \pm}
$$

which, in components, is rewritten as follows

$$
\pm \mathrm{i} \mathcal{H}_{ \pm \mid i j k}=\frac{1}{3 !} \epsilon_{i j k \ell m n} \mathcal{H}_{ \pm}^{\ell m n}
$$

Eq.(3.37) implies that:

$$
\begin{aligned}
T_{j}^{i}[\mathcal{H}] & =-\frac{9}{16}\left(\mathcal{H}_{+}{ }^{i \cdot} \mathcal{H}_{-\mid j .}+\mathcal{H}_{-}{ }^{i \cdot \cdot} \mathcal{H}_{+\mid j \cdot .}-\frac{1}{3} \delta_{j}^{i} \mathcal{H}_{-}^{\cdots} \mathcal{H}_{+\mid \cdots}\right) \\
& =-\frac{3}{32} \delta_{j}^{i} \mathcal{H}_{-}^{\cdots} \mathcal{H}_{+\mid \cdots} \\
& =\frac{1}{32} H^{-3 / 2} \partial \gamma_{I} \bar{\partial} \bar{\gamma}_{J} \Delta^{I J} \delta_{j}^{i}
\end{aligned}
$$

where we have used the result:

$$
\begin{aligned}
\mathcal{H}_{+} \wedge \star \mathcal{H}_{-} & =-8 H^{-1} \partial \gamma_{I} \bar{\partial} \bar{\gamma}_{J} \Delta^{I J} \Omega_{A L E} \wedge \Omega_{\mathbb{R}^{1,3}} \wedge \Omega_{\mathbb{R}^{2}} \\
& \equiv 3 ! \mathcal{H}_{+}^{\cdots} \mathcal{H}_{-\mid} \ldots \times \text { Vol }
\end{aligned}
$$

having named the complete volume form of the ten-dimensional space-time:

$$
\mathrm{Vol}=H^{1 / 2} \Omega_{\mathbb{R}^{1,3}} \wedge \Omega_{A L E} \wedge \Omega_{\mathbb{R}^{2}}=\sqrt{-\operatorname{det} g} d X^{0} \wedge \ldots \wedge d X^{9}
$$

Inserting eq.s (3.35), (3.38) and (3.9) into Einstein's eq.(2.9):

$$
\begin{aligned}
R_{b}^{a} & =T_{b}^{a}\left[F_{[5]}^{R R}\right]-\frac{3}{16} \delta_{b}^{a} \mathcal{H}_{+}^{\cdots} \mathcal{H}_{-\mid \cdots} \\
R_{j}^{i} & =T_{j}^{i}\left[F_{[5]}^{R R}\right]+T_{j}^{i}[\mathcal{H}]
\end{aligned}
$$


we see that they are satisfied, provided

$$
\alpha=1
$$

and the master equation (3.27) is satisfied. This concludes our proof that an exact 3-brane solution with a non-trivial flux through a homology 2-cycle of a generic smooth ALE manifold, does indeed exist. In the next subsection we prove that this bulk solution admits two Killing spinors and is therefore a BPS state with $\mathcal{N}=2$ preserved supersymmetry.

We would like to end this section by stressing a crucial difference between our solution and the "conifold" case (see [14] for related discussions). In the former, the $S^{2}$ cycles exist only near the apex and thus we do not have a 3-form flux at infinity, whereas in the latter, the base of the cone supports such cycles and, as a result, the flux at infinity is non-vanishing?

\subsection{Proof of bulk supersymmetry}

As usual, in order to investigate the supersymmetry properties of the bosonic solution we have found it suffices to consider the supersymmetry transformation of the fermionic fields (the gravitino and the dilatino) and impose that, for a Killing spinor, they vanish identically on the chosen background. By using the formulation of [32], one easily gets:

$$
\begin{aligned}
\delta \psi_{M}= & \mathcal{D}_{M} \chi+\frac{1}{16} \mathrm{i} \Gamma^{A_{1} \ldots A_{5}} F_{A_{1} \ldots A_{5}} V_{M}^{B} \Gamma_{B} \chi \\
& +\frac{1}{32}\left(-\Gamma_{B A_{1} \ldots A_{3}} V_{M}^{B}+9 \Gamma^{A_{1} A_{2}} V_{M}^{A_{3}}\right) \mathcal{H}_{+\mid A_{1} A_{2} A_{3}} \chi^{\star} \\
\delta \lambda= & -\mathrm{i} \frac{1}{8} \Gamma^{A_{1} A_{2} A_{3}} \mathcal{H}_{+\mid A_{1} A_{2} A_{3}} \chi
\end{aligned}
$$

where the supersymmetry parameter $\chi$ is a complex ten-dimensional Weyl spinor:

$$
\Gamma_{11} \chi=\chi
$$

and where we have already used the information that on our background the dilaton and the Ramond scalar vanish. Using the gamma matrix basis described in appendix $\mathrm{A}$ (see in particular eq.s (A.7, A.8,A.9) and writing the 32-component spinor $\chi$ as a tensor product:

$$
\chi=\varepsilon \otimes \eta
$$

of a 4-component spinor $\varepsilon$, related to the 3-brane world volume with an 8-component spinor $\eta$ related to the transverse manifold $M_{6}$, the dilatino transformation rule takes the following form:

$$
\delta \lambda=-\mathrm{i} \frac{1}{8} \gamma_{5} \varepsilon \otimes \tau^{i j k} \mathcal{H}_{+\mid i j k} \eta
$$

Hence the first condition a Killing spinor must satisfy comes from the vanishing of the dilatino rule and reads as follows:

$$
\Pi(\mathcal{H}) \eta \equiv \tau^{i j k} \mathcal{H}_{+\mid i j k} \eta=0
$$

Using the self-duality relation (3.37) we can immediately verify that:

$$
\Pi(\mathcal{H})=\Pi(\mathcal{H}) \frac{1}{2}\left(1+\mathrm{i} \tau_{\bullet}\right)
$$

so that in order to solve eq. (3.47) it suffices to impose the $M_{6}$ chirality choice:

$$
\text { i } \tau_{\bullet} \eta=-\eta
$$

\footnotetext{
${ }^{6}$ We thank I. Klebanov for clarifying this point.
} 
Since $\Gamma_{11}=-\mathrm{i} \gamma_{5} \otimes \tau_{\bullet}$, eq. (3.49) implies:

$$
\gamma_{5} \varepsilon=\varepsilon
$$

Inserting conditions (3.49) and (3.50) into the gravitino transformation rule, by use of the specific structure of $F_{A_{1} \ldots A_{5}}$ defined by eq.(3.33), we obtain two new conditions that must be imposed on the Killing spinor:

$$
\begin{aligned}
0 & =\mathcal{D}_{a} \chi-\frac{1}{8} f_{i} \gamma_{a} \otimes \tau^{i} \chi-\frac{1}{32} \gamma_{5} \gamma_{a} \Pi(\mathcal{H}) \chi^{\star} \\
0 & =\mathcal{D}_{j} \chi-\frac{1}{8} f_{i} \tau^{i} \tau_{j} \chi+\frac{1}{32} \tau_{j} \Pi(\mathcal{H}) \chi^{\star}
\end{aligned}
$$

In the above equations $f_{i}$ is the intrinsic derivative of the harmonic function $H$ defined by eq. (3.34). Recalling the form of the spin connection on our background (see eq.(3.8)) we easily verify that eq. (3.51) reduces to:

$$
\partial_{a} \varepsilon \otimes \eta-\frac{1}{32} \gamma_{5} \gamma_{a} \varepsilon^{\star} \otimes \Pi(\mathcal{H}) \eta^{\star}=0
$$

This equation is satisfied if we choose constant supersymmetry parameters on the 4-dimensional world volume:

$$
\partial_{a} \varepsilon=0
$$

and imposing the further condition

$$
\Pi(\mathcal{H}) \eta^{\star}=0
$$

on the transverse commuting spinor $\eta^{\star}$.

Taking condition (3.55) into account and recalling once again the form of the spin connection (3.8), eq. 3.52 becomes:

$$
0=\varepsilon \otimes\left(\widehat{\mathcal{D}}+\frac{1}{8} H^{-1} \widehat{d} H\right) \eta
$$

where the hatted operators $\widehat{\mathcal{D}}$ and $\widehat{d}$ are the covariant and ordinary exterior derivatives on $M_{6}=\mathbb{R}^{2} \times$ ALE considered by itself without the warp factor $H(z, \bar{z}, y)$.

Introducing a rescaling factor $H^{-1 / 8}(z, \bar{z}, y)$ and a further tensor product splitting of the 8 component spinor

$$
\eta=H^{-1 / 8}(z, \bar{z}, y) \theta \otimes \xi
$$

into a 2 -component spinor $\theta$ relative to the manifold $\mathbb{R}^{2}$ times a 4 -component spinor $\xi$ living on the ALE manifold, eq. (3.56) reduces to the condition of covariant constancy on ALE:

$$
0=\widehat{\mathcal{D}} \xi
$$

Collecting all the conditions we have found and using the gamma matrix basis described in eq.s (A.10) of the appendix we can conclude that our background admits as many 4-dimensional supersymmetries on the world-volume of the 3 brane as there are simultaneous solutions of the following three equations:

$$
\begin{aligned}
\theta \otimes \xi & =\sigma_{3} \theta \otimes \bar{\gamma}_{5} \xi \\
0 & =\partial \gamma_{I}(z)\left(\sigma^{1}-\mathrm{i} \sigma^{2}\right) \theta^{\star} \otimes \omega_{u v}^{I} \bar{\gamma}^{u v} \xi^{\star} \\
0 & =\widehat{\mathcal{D}} \xi
\end{aligned}
$$

where $\omega_{u v}^{I}$ are the intrinsic components of the anti-self-dual normalizable 2-forms on ALE. 
So far we have not used the specific geometric properties of the ALE manifold. It is at this stage they come into the game, playing an essential role. Squaring eq. 3.61) we obtain the integrability condition

$$
-\frac{1}{4} R^{u v} \bar{\gamma}_{u v} \xi=0
$$

where $R^{u v} \bar{\gamma}_{u v}$ is the curvature 2-form of the ALE manifold. This latter is HyperKähler and as such it has a triplet of covariantly constant self-dual 2-forms $\Omega^{x},(x=1,2,3)$ whose intrinsic components satisfy the quaternionic algebra. This implies that the holonomy of the manifold is $\mathrm{SU}(2)_{L}$ rather than $\mathrm{SO}(4)=\mathrm{SU}(2)_{\mathrm{L}} \times \mathrm{SU}(2)_{\mathrm{R}}$ and that the curvature 2-form is anti-selfdual. This follows from the integrability condition for the covariant constancy of the self-dual HyperKähler 2-forms. On the other hand, from the Hirzebruch signature $\tau$ of the ALE manifold it follows that there are exactly $\tau$ normalizable anti-self-dual forms $\omega_{I}$. From the trivial gamma matrix identity:

$$
\bar{\gamma}_{5} \bar{\gamma}_{u v}=-\frac{1}{2} \epsilon_{u v s t} \bar{\gamma}^{s t}
$$

follows that the two chirality eigenspaces:

$$
\bar{\gamma}_{5} \xi_{ \pm}= \pm \xi_{ \pm}
$$

are respectively annihilated by the contraction of $\bar{\gamma}_{u v}$ with any self-dual or anti self-dual 2-form. Therefore it suffices to choose:

$$
\xi=\xi_{+}
$$

and equations (3.60), (3.62) are simultaneously satisfied. Eq. (3.59) is satisfied by setting:

$$
\sigma_{3} \theta=\theta
$$

and once the integrability condition (3.62) is fulfilled eq. (3.61) can be integrated yielding two linear independent solutions $\xi_{+}^{1,2}$ spanning the irreducible representation $(0,1 / 2)$ of $\mathrm{SO}(4)$. The other irreducible representation $(1 / 2,0)$ corresponds to spinors that are not Killing and do not generate supersymmetries preserved by the background. In conclusion we have 2 Killing spinors generating an $\mathcal{N}=2$ supersymmetry on the world volume. In other words the bosonic background we have constructed corresponds to a BPS state preserving a total of $2 \times 4=8$ supercharges.

\section{The Eguchi-Hanson case}

As showed in the previous sections, the complete integration of the supergravity field equations is reduced to the solution of a single differential equation, namely eq. (3.27). In this section we investigate the properties of such an equation, choosing the simplest instance of an ALE manifold, namely the Eguchi-Hanson space [36], which in the ADE classification corresponds to $A_{1} \sim \mathbb{Z}_{2}$ (see appendix $\mathrm{B}$ for a more detailed treatment of the Eguchi-Hanson space and for the definition of the relevant conventions). While in this section we will go through a somewhat detailed mathematical analysis, section 5 contains a summary of the most relevant results and their physical implications. It should be said from the beginning that, whereas section 3 is entirely based on type IIB supergravity, in the following we are going to make some (we believe resonable) assumptions on the nature of the microscopic theory, namely the structure of the source terms needed to fix the boundary conditions. 
The Eguchi-Hanson metric has the form:

$$
\begin{aligned}
d s_{E H}^{2} & =g(r)^{-2} d r^{2}+r^{2}\left(\sigma_{x}^{2}+\sigma_{y}^{2}\right)+r^{2} g(r)^{2} \sigma_{z}^{2} \\
g(r)^{2} & =1-\left(\frac{a}{r}\right)^{4}
\end{aligned}
$$

where $r \geq a, \theta \in\left[0, \pi\left[\right.\right.$ and $\psi, \phi \in\left[0,2 \pi\left[\right.\right.$. The 1 -forms $\sigma^{i}=\left\{\sigma^{x}, \sigma^{y}, \sigma^{z}\right\}$ are listed in eq. (B.2) of appendix B.

This space has a unique homology 2-cycle $\Sigma$ located at $r=a$ and spanned by the coordinates $\theta, \phi$. The anti-self dual form $\omega$ fulfilling eqs. (3.19) is:

$$
\omega=\frac{a^{2}}{2 \pi} d\left(\frac{\sigma_{z}}{r^{2}}\right)
$$

The function $\Delta(y)$ defined in $(3.19)$ is explicitly evaluated to be:

$$
\Delta(y)=\frac{2 a^{4}}{\pi^{2} r^{8}}
$$

The equation for $H$ in the Eguchi-Hanson case can then be easily obtained from the general expression in eq.(3.27). As it is usually the case, we make a spherically symmetric ansatz, compatible with the background at hand: for the case we shall be interested in, the coefficients of the equation for $H$ depend only on the radial coordinates $\rho=\sqrt{z \bar{z}}$ and $r$ on $\mathbb{R}^{2}$ and on the Eguchi-Hanson space respectively, and we can then assume the same property for $H$ to hold and write it as $H(\rho, r)$ ]. The equation for $H$ can then be written as:

$$
\left(\partial_{4}^{2}+\partial_{5}^{2}\right) H+\frac{1}{r^{3}} \partial_{r}\left(r^{3} g(r)^{2} \partial_{r} H\right)=-\frac{2 a^{4}}{\pi^{2} r^{8}}\left|\partial_{z} \gamma\right|^{2}+\mathcal{S}(\rho, r)
$$

where $\mathcal{S}(\rho, r)$ is a source term for the 3 -brane charge for which we also make a spherical ansatz. Differently from the first term on the right hand side, $\mathcal{S}(\rho, r)$ is not deduced from the dynamics in the bulk. In the present analysis its presence should be intended only for the sake of fixing boundary conditions near the cycle and it will be determined in the following sections.

\subsection{Solution method by partial Fourier transform}

The method we use to study eq. (4.4) is based on a partial Fourier transform on the coordinates of the flat $\mathbb{R}^{2}$ space that reduces our problem to that of a massive Laplace equation on the residual four-dimensional manifold in the unknown function $\widetilde{H}(\mu, r)$, Fourier transform in $\mathbb{R}^{2}$ of $H(\rho, r)-1$ (with $\mu$ being the modulo of the momentum in $\mathbb{R}^{2}$ ). We refer for the details of the analysis to appendix $\mathrm{Q}$. As we are going to show this method allows a parallel treatment of our case $\mathbb{R}^{2} \times \mathrm{ALE}_{\mathrm{EH}}$ with the case $\mathbb{R}^{2} \times \mathbb{R}^{4}$ which is the covering space for fractional D3 branes on orbifolds [7]. Such a parallelism is very instructive since the comparison with the flat case provides the necessary insight to discuss and interpret the appropriate boundary conditions. The main goal of the analysis in the following sub-sections is to determine $H(\rho, r)$ through its near-cycle and far-from-cycle asymptotic limits and an exact analytical description through a power series expansion of $H$ in an open neighborhood of the cycle, consistently with the boundary conditions. The reason why we choose to study the equation in the Fourier transform

\footnotetext{
${ }^{7}$ We shall consider the case in which $\gamma(z)=\log (z)$. In a more general situation $|\partial \gamma(z)|^{2}$ could also depend on the angular coordinate on $\mathbb{R}^{2}$. In this case the function $H$ would have an angular dependence in $\mathbb{R}^{2}$ as well.
} 
$\widetilde{H}(\mu, r)$ of $H(\rho, r)-1$ instead of $H(\rho, r)$ is a matter of convenience in dealing with the boundary conditions at infinity: at large distances $H(\rho, r) \rightarrow 1$ and therefore $\widetilde{H}(\mu, r) \rightarrow 0$. The relation between $\widetilde{H}(\mu, r)$ and $H(\rho, r)$ is deduced from the standard definition of Fourier transform given in appendix $\mathrm{Q}$ and reads:

$$
\widetilde{H}(\mu, r)=\frac{1}{2 \pi} \int_{0}^{\infty} d \rho \rho J_{0}(\mu \rho)(H(\rho, r)-1)
$$

The equation for $\widetilde{H}(\mu, r)$ has the following form:

$$
L_{r}[a, \mu] \widetilde{H}(\mu, r)=J(\mu, r)
$$

where we have introduced the second order differential operator

$$
L_{r}[a, \mu] u=\frac{1}{r^{3}} \frac{d}{d r}\left(r^{3} g(r)^{2} \frac{d u}{d r}\right)-\mu^{2} u
$$

and where we have defined the source function:

$$
J(\mu, r) \equiv-\frac{2 a^{4}}{\pi^{2} r^{8}} j(\mu)+S(\mu, r)
$$

$j(\mu)$ denoting the Fourier transform of $|\partial \gamma(z)|^{2}$ and $S(\mu, r)$ being the transform of the source term $\mathcal{S}(\rho, r)$ for the 3-brane charge.

The notation $L_{r}[a, \mu]$ is very convenient since it encodes various cases into a single formula. For $a=0, L_{r}[0, \mu]$ is just the massive radial Laplacian on flat $\mathbb{R}^{4}$ space. Alternatively for $\mu=0$, $L_{r}[a, 0]$ is the massless radial Laplacian on either $\mathrm{ALE}_{\mathrm{EH}}$ or $\mathbb{R}^{4}$ depending on the value of $a$. By massive or massless Laplacian we mean the operator $\left(\square-\mu^{2}\right)$ with $\mu^{2}>0$ or $\mu^{2}=0$.

It is known from the standard theory of differential equations that the general integral $\widetilde{H}(\mu, r)$ of eq. (4.6) has the form:

$$
\widetilde{H}(\mu, r)=\beta_{1} u_{1}(\mu, r)+\beta_{2} u_{2}(\mu, r)+u_{n-h}(\mu, r)
$$

where $u_{1,2}(\mu, r)$ are two independent solutions of the homogeneous equation associated with (4.6):

$$
L_{r}[a, \mu] u=0
$$

while $u_{n-h}(\mu, r)$ denotes a particular solution of the non-homogeneous equation. The constants $\beta_{1}$ and $\beta_{2}$ are fixed by the boundary conditions.

It is useful to express the unique solution of eq.4.6) fulfilling the required boundary conditions in terms of a Green function $G\left(a, \mu \mid r, r^{\prime}\right)$ :

$$
\widetilde{H}(\mu, r)=\int_{a}^{\infty} G\left(a, \mu \mid r, r^{\prime}\right) J\left(\mu, r^{\prime}\right)\left(r^{\prime}\right)^{3} d r^{\prime}
$$

where the Green function is defined by the equation:

$$
L_{r}[a, \mu] G\left(a, \mu \mid r, r^{\prime}\right)=\frac{\delta\left(r-r^{\prime}\right)}{\left(r^{\prime}\right)^{3}}
$$

The defining properties of $G\left(a, \mu \mid r, r^{\prime}\right)$ are recalled and thoroughly discussed in appendix $\mathbf{Q}$. The Green function associated with the homogeneous equation (4.10) is generally expressed in terms of a combination of the solutions $u_{1,2}$. The main problem to be dealt with in the next sections is to determine, on one hand, the source term $S(\mu, r)$, and, on the other hand, the form of the Green function such that it implements the required physical boundary conditions of the problem. These are conditions on the behavior of $\widetilde{H}$ near the cycle $r \rightarrow a$ and at infinity, $r \rightarrow \infty$. 


\subsection{Asymptotics of the the two independent solutions $u_{1}, u_{2}$ for the homoge- neous equation}

So far our discussion of the differential equation has been fully general and applies to both $\mathbb{R}^{4}$ and to the Eguchi-Hanson case. We turn next to a more detailed consideration of the homogeneous equation and of its basis of solutions in various sub-cases. Indeed the parameter space of our differential operator, spanned by $a$ and $\mu$ is partitioned into four sub-cases according to the following scheme:

\begin{tabular}{|c|c|c|}
\hline & $a=0$ & $a \neq 0$ \\
\hline$\mu=0$ & massless $\mathbb{R}^{4}$ case & massless $E H$ case \\
\hline$\mu \neq 0$ & massive $\mathbb{R}^{4}$ & massive $E H$ case \\
\hline
\end{tabular}

In three of the above four sectors the homogeneous equation can be solved in terms of elementary transcendental or special functions so that all asymptotic behaviors are easily calculated. The really hard corner is the lowest right one, namely the massive Eguchi-Hanson case. As we show in the next section, for $\mu \neq 0, a \neq 0$ the homogeneous equation (4.6) is a particular instance of the confluent Heun differential equation and its solutions can be expressed in terms of a power series. The same pattern of asymptotic behavior is reproduced also in this case and provides the needed guideline to fix the boundary conditions.

Let us write down the basis of solutions in the three cases where they are easily accessible by elementary methods and indicate our nomenclature for the massive EH case that we address in the following section:

$$
\begin{array}{llllc}
a=0 ; \mu=0 & \underline{\text { massless } \mathbb{R}^{4}} u_{1}(0,0, r) & \frac{1}{r^{2}} \\
u_{2}(0,0, r) & = & 1 \\
a=0 ; \mu \neq 0 ; & \underline{\text { massive } \mathbb{R}^{4}} & u_{1}(0, \mu, r) & = & \frac{\mu}{r} K_{1}(\mu r) \\
u_{2}(0, \mu, r) & = & \frac{2}{\mu r} I_{1}(\mu r) \\
a \neq 0 ; \mu=0 & \underline{\text { massless } E H} & u_{1}(a, 0, r) & = & -\frac{1}{2 a^{2}} \log \left(\frac{r^{2}-a^{2}}{r^{2}+a^{2}}\right) \\
& & u_{2}(a, 0, r) & = & 1 \\
a \neq 0 ; \mu \neq 0 & \underline{\text { massive } E H} & u_{1}(a, \mu, r) & = & -\frac{1}{2 a^{2}} H^{(I)}(\mu, r) \\
& & u_{2}(a, \mu, r) & = & H^{(R)}(\mu, r)
\end{array}
$$

where $K_{\nu}(z)$ and $I_{\nu}(z)$ denote Bessel function of index $\nu$ and type $K$ or $I$, respectively. The reader can easily check that the above solutions have been normalized in such a way as to fulfill the normalization (C.18) of the Wronskian. The notation $H^{(I)}$ and $H^{(R)}$ is reserved for the confluent Heun functions, to be discussed in the next section, that have the corresponding behavior at $r \rightarrow a$ as their analogues in the massless case. To understand the rationale of the previous statement it is instructive to consider the series expansion of $u_{1,2}(0, \mu, r)$ at $r=0$. We find:

$$
\begin{aligned}
& u_{1}(0, \mu, r) \simeq{ }_{r \rightarrow 0} \frac{1}{r^{2}}+\frac{\mu^{2}}{4}\left(-1+2 \gamma+2 \log \left(\frac{r \mu}{2}\right)\right)+\mathcal{O}\left(r^{2}\right) \\
& u_{2}(0, \mu, r) \simeq{ }_{r \rightarrow 0} 1+\frac{\mu^{2} r^{2}}{8}+\mathcal{O}\left(r^{4}\right)
\end{aligned}
$$


where $\gamma=0.577216$ is the Euler gamma number. As one sees the leading behavior of the massive solutions for $r \rightarrow 0$ is just identical with the behavior of the massless solutions, $u_{1}$ is singular and has a second order pole $1 / r^{2}$ while $u_{2}$ is a regular power series and tends to 1 . Indeed choosing the overall normalization consistent with eq. (C.18) we could also arrange for the coefficients of the pole and of the constant term in $u_{1}$ and $u_{2}$ respectively, to be equal to one. In the case of the regular solution $u_{2}(0, \mu, r)$ the generic coefficient of the power series expansion in eq.4.15) can be easily calculated and we have:

$$
u_{2}(0, \mu, r)=\sum_{n=0}^{\infty} \frac{1+n}{4^{n}((1+n) !)^{2}}(\mu r)^{2 n}
$$

As we see in the next section a similar regular series, although much more complicated can be written down for $u_{2}(a, \mu, r) \equiv h^{r e g}(a, \mu, r)$. Let us next consider the behavior at $r=a$ of the massless solution $u_{2}(a, 0, r)$. We have

$$
u_{1}(a, 0, r) \simeq{ }_{r \rightarrow a} \frac{-1}{2 a^{2}} \log \left(\frac{r-a}{a}\right)+\mathcal{O}(r-a)
$$

As we see the double pole singularity $1 / r^{2}$ appearing in the $\mathbb{R}^{4}$ case has been replaced by a logarithmic singularity $\log (r-a)$ appearing in the Eguchi-Hanson case. What remains identical in the two cases is the relation between the massive and massless solutions. Also for $\mu \neq 0$ we find in the next section that:

$$
\begin{aligned}
& u_{1}(a, \mu, r) \simeq{ }_{r \rightarrow a} \frac{-\log (r-a)}{2 a^{2}}+\text { reg. } \\
& u_{2}(a, \mu, r) \simeq{ }_{r \rightarrow a} 1+\mathcal{O}(r-a)
\end{aligned}
$$

Using this information on the asymptotic behavior of the two fundamental solutions we can complete our program of constructing the Green function by imposing the boundary conditions of physical relevance.

To this effect we need the preliminary analysis of the near-cycle limit in the Eguchi-Hanson space. Let us go back to the Eguchi-Hanson metric in the form (4.1) and let us perform the following change of variable:

$$
r \rightarrow v=\frac{\sqrt{r^{4}-a^{4}}}{2 r}
$$

The form of the metric near the cycle $(r \rightarrow a)$ can now be calculated by expanding the metric (4.1) in power series of $v$ at $v=0$. We obtain:

$$
d s_{E H}^{2} \simeq_{v \rightarrow 0} \quad d v^{2}+v^{2} d \psi^{2}+\frac{a^{2}}{4}\left(d \theta^{2}+d \phi^{2} \sin ^{2} \theta\right)+\mathcal{O}\left(v^{4}\right)
$$

showing that near the homology cycle the Eguchi-Hanson metric approximates that of a manifold $\mathbb{R}^{2} \times \mathrm{S}^{2}$.

In view of this result we consider the transcription of the homogeneous radial equation to the new variable $v$, defined by eq.(4.19). We obtain, for small $v$ :

$$
\frac{d^{2}}{d v^{2}} \widetilde{H}+\frac{1}{v} \frac{d}{d v} \widetilde{H}-\mu^{2} \widetilde{H}=0
$$

admitting the following two linearly independent solutions:

$$
\bar{u}_{1}(v, \mu)=\frac{1}{a^{2}} K_{0}(v \mu) \quad ; \quad \bar{u}_{2}(v, \mu)=I_{0}(v \mu)
$$


whose behavior for $v \rightarrow 0$ is:

$$
\begin{aligned}
& \bar{u}_{1}(v, \mu) \simeq_{v \rightarrow 0} \quad-\frac{\log (v)}{a^{2}}+\text { reg } \\
& \bar{u}_{2}(v, \mu) \simeq_{v \rightarrow 0} \quad 1+\frac{\mu^{2} v^{2}}{4}+\mathcal{O}\left(v^{3}\right)
\end{aligned}
$$

If in eq.s (4.23) we reinsert the original variable $r$ via the relation (4.19) we obtain the announced proof that, near the cycle, the leading behavior of the massive solutions $u_{1}(a, \mu, r)$ is the same as the behavior of the corresponding massless solutions. By such a substitution we get:

$$
\begin{aligned}
& u_{1}(a, \mu, r) \simeq_{r \rightarrow a} \bar{u}_{1}\left(\frac{\sqrt{(r-a)}}{a}, \mu\right)=-\frac{1}{2 a^{2}} \log (r-a)+\text { reg } \\
& u_{2}(a, \mu, r) \simeq_{r \rightarrow a} \quad \bar{u}_{2}\left(\frac{\sqrt{(r-a)}}{a}, \mu\right)=1+\mathcal{O}(r-a)
\end{aligned}
$$

\subsection{The 3-brane charge, the physical boundary conditions and the final form of the Green function}

Equipped with the results of the previous section, let us now consider the case of a 3-brane with vanishing 3-form placed either at the origin of $\mathbb{R}^{6}$ in the orbifold case where the transverse space is $M_{6}=\mathbb{R}^{2} \times \mathbb{R}^{4} / \Gamma$ or at the homology cycle $r=a$ in the case where the transverse space is $M_{6}=\mathbb{R}^{2} \times \mathrm{ALE}_{\mathrm{EH}}$. Naming $Q$ the charge of such a brane, the expected behavior of the $H$ function near the brane is, in the two cases, the following one:

$$
H \simeq \frac{Q}{\left(x_{4}^{2}+x_{5}^{2}+\ldots+x_{9}^{2}\right)^{2}} \quad \text { for } \mathbb{R}^{6} ; \quad H \simeq \frac{Q / 2 a^{2}}{\left(\rho^{2}+v^{2}\right)} \quad \text { for } \mathbb{R}^{2} \times \mathrm{ALE}
$$

The first of eq.s (4.25) is obvious. The second is due to the discussion of the previous section. Near the cycle the Laplacian on $M_{6}$ becomes:

$$
\square_{M_{6}} \sim \square_{\mathbb{R}^{4}}+\square_{S^{2}}
$$

and since $S^{2}$ is a compact, positive curvature manifold there are no zero modes of $\square_{S^{2}}$ except the constant. Therefore the non trivial part of $H$ behaves as a harmonic function on $\mathbb{R}^{4}$, namely the second of eq.s (4.25). The scale factor $1 /\left(a^{2}\right)$ appearing there is understood as follows. If $Q$ is the total charge perceived at infinity, the density of charge on the homology 2-sphere of radius $\frac{a}{2}$ is:

$$
q=\frac{Q}{a^{2} \pi}
$$

Hence what appears as charge in the near cycle $\mathbb{R}^{4}$ plane is $q$ rather than $Q$. Finally the $\pi / 2$ factor that is needed to match the second with the first of equations (4.25) is just a matter of convenient normalization. Let us now perform the Fourier-Bessel transform of eq.s (4.25). We obtain

$$
\begin{aligned}
\frac{1}{2 \pi} \int_{0}^{\infty} J_{0}(\mu \rho) \rho \frac{Q}{\left(r^{2}+\rho^{2}\right)^{2}} d \rho & =\frac{Q}{4 \pi} \frac{\mu}{r} K_{1}(\mu r)=\frac{Q}{4 \pi} u_{1}(0, \mu, r) \\
\frac{1}{2 \pi} \int_{0}^{\infty} J_{0}(\mu \rho) \rho \frac{Q}{2 a^{2}\left(v^{2}+\rho^{2}\right)} d \rho & =\frac{Q}{4 \pi} \frac{1}{a^{2}} K_{0}(\mu v)=\frac{Q}{4 \pi} \bar{u}_{1}(v, \mu)
\end{aligned}
$$

The important conclusion implied by the above analysis is that, in terms of the fundamental basis of solutions $u_{1}, u_{2}$ we have obtained the physically appropriate boundary conditions for 
the function $\widetilde{H}(\mu, r)$. In both the orbifold or smooth ALE case, in the limits $r \rightarrow 0$ and $r \rightarrow a$ respectively, we have:

$$
\widetilde{H}(\mu, r) \sim \frac{Q}{4 \pi} u_{1}^{d i v}(\mu, r)+\mathrm{reg}
$$

where $Q$ is the 3-brane charge and $u_{1}^{d i v}(\mu, r)$ denotes the divergent part of the solution $u_{1}(a, \mu, r)$ defined in eq.s(4.13). This condition, together with the boundary condition at infinity, fixes the coefficients $\beta_{1}, \beta_{2}$ in eq. (4.9) to be:

$$
\beta_{1}=\frac{Q}{4 \pi} \quad ; \quad \beta_{2}=0
$$

The appropriate expression for the Green function in order for it to implement the physical boundary conditions defined above is derived in appendix $\bar{Q}$ and is the following:

$$
G\left(r, r^{\prime}\right)= \begin{cases}-\frac{1}{2} u_{2}(r) u_{1}\left(r^{\prime}\right) & \text { for } r<r^{\prime} \\ -\frac{1}{2} u_{1}(r) u_{2}\left(r^{\prime}\right) & \text { for } r>r^{\prime}\end{cases}
$$

Acting with such a Green function on a source:

$$
S(\mu, r)=-\frac{Q}{2 \pi} \frac{\delta(r-a)}{r^{3}}
$$

we obtain a solution with the prescribed behavior as in eq. (4.29). From the physical viewpoint the source (4.32) comes from the world-volume action of the 3-brane.

\subsection{Reduction to the confluent Heun equation}

As we have seen in previous sections a convenient approach to the solution of equation (4.4) relies on the partial Fourier transform of eq.(C.1) leading to the new eq.s (4.6), (4.7) for the function $\widetilde{H}(\mu, r)$. So far we have analyzed such an equation from a general viewpoint and we have discussed how to impose the correct boundary conditions from the asymptotic behavior of the two solutions $u_{1}$ and $u_{2}$ of the associated homogeneous equation. In this section we eventually come to a more in depth analysis of the equation in a generic point of its moduli space, i.e. at $\mu \neq 0, a \neq 0$.

Parameterizing the radial direction in the Eguchi-Hanson space through the new variable $w=\left(a^{2}-r^{2}\right) /\left(2 a^{2}\right)$, equation (4.6), (4.7) can be rewritten in the following form:

$$
\begin{aligned}
& w^{2} \frac{d^{2} \widetilde{H}}{d w^{2}}+w P_{1}(w) \frac{d \widetilde{H}}{d w}+P_{2}(\mu, w) \widetilde{H}=\frac{h(\mu) w}{(1-w)(1-2 w)^{3}} \\
& \widetilde{H}(\mu, w) \stackrel{w \rightarrow-\infty}{\longrightarrow} 0
\end{aligned}
$$

where

$$
\begin{aligned}
& P_{1}(w)=\frac{2 w-1}{w-1} \quad ; \quad P_{2}(\mu, w)=k w\left(\frac{2 w-1}{w-1}\right) \\
& h(\mu)=\frac{1}{2 \pi^{2} a^{2}} j(\mu)=\frac{1}{2 \pi^{2} a^{2}} \int_{0}^{\infty} \frac{d^{2} x}{(2 \pi)^{2}}\left|\partial_{z} \gamma(z)\right|^{2} e^{\mathrm{i} \vec{p} \cdot \vec{x}} ; \quad k=\frac{\mu^{2} a^{2}}{4}
\end{aligned}
$$

We have omitted in the source term the part proportional to $S(\mu, r)$ since, as explained in the previous sections, its effect amounts in a determination of the relative coefficients of the two independent solutions $u_{1,2}$ of the homogeneous equation, as in eq. (4.30). We postpone to 
appendix a more detailed analysis of eq. (4.33), as a non-homogeneous confluent Heun equation, and of its solution. In the present section we shall determine the analytical expression of $\widetilde{H}$ in a finite neighborhood of the cycle, represented by the regular singularity $w=0$ and implement on it the the required boundary conditions: the behavior of the integral at infinity, defined in eq. (4.33), and its behavior at $w \rightarrow 0$, determined by the presence of source terms as we explained in section 4.3. Finally, using these exact results we shall compute the anti-Fourier transform $H$ of $\widetilde{H}$ in its asymptotic near-cycle limit, confirming the predictions made in the previous section.

Let us now discuss the solution around $w=0$ and the asymptotic solution for $w \rightarrow-\infty$. The expansion of the solution around $w=0$ corresponds to considering its behavior near the exceptional cycle: $r \sim a$. Since $w=0$ is a regular singularity there are standard ways to determine the power series expansion of the the solutions for both the homogeneous [37] and non-homogeneous equations around such a point.

First let us focus on the homogeneous equation associated with (4.33) and let us denote it symbolically by $L_{w} \widetilde{H}=0$. This equation has two independent solutions, one being regular at $w=0$ will be denoted by $H^{(R)}(\mu, w)$, the other having a logarithmic behavior near the same point will be denoted by $H^{(I)}(\mu, w)$. From standard analysis we may determine the power series expansions of the above integrals around $w=0$ :

$$
\begin{aligned}
H^{(R)}(\mu, w) & =\sum_{n=0}^{\infty} c_{n}^{R}(\mu) w^{n}=\sum_{n=0}^{\infty} c_{n}(\mu, \sigma=0) w^{n} \\
H^{(I)}(\mu, w) & =\lim _{\sigma \rightarrow 0} \frac{d}{d \sigma}\left(w^{\sigma} \sum_{n=0}^{\infty} c_{n}(\mu, \sigma) w^{n}\right)
\end{aligned}
$$

the coefficients $c_{n}(\mu, \sigma)$ will be derived in appendix $\mathrm{C}$ and have the form:

$$
\begin{aligned}
& c_{n}(\mu, \sigma)=(-)^{n} c_{0}(\mu) \frac{F_{n}(\mu, \sigma)}{\prod_{j=1}^{n} f_{0}(\sigma+j)}=(-)^{n} c_{0}(\mu) \frac{F_{n}(\mu, \sigma)}{\prod_{j=1}^{n}(\sigma+j)^{2}} \\
& F_{n}(\mu, \sigma)=\operatorname{det} \mathbb{F}_{n}(\mu, \sigma)
\end{aligned}
$$

where $\mathbb{F}_{n}(\mu, \sigma)$ is an $n \times n$ matrix, see appendix $\mathbb{C}$, whose entries are:

$$
\begin{aligned}
{\left[\mathbb{F}_{n}(\mu, \sigma)\right]_{i i} } & =k-\sigma-n+i ; \quad\left[\mathbb{F}_{n}(\mu, \sigma)\right]_{i j>i}=-(k+\sigma+n-j) \\
{\left[\mathbb{F}_{n}(\mu, \sigma)\right]_{i+1 i} } & =(\sigma+n-i)^{2} ; \quad i, j=1, \ldots, n
\end{aligned}
$$

Both the series for $H^{(I)}(\mu, w)$ and $H^{(R)}(\mu, w)$ converge for $|w|<1$, namely for $r^{2}-a^{2}<2 a^{2}$. A generic solution of the homogeneous equation will be expressed as a linear combination of $H^{(I)}(\mu, w)$ and $H^{(R)}(\mu, w)$, the relative coefficients will be determined in quite the same way as the constants $\beta_{1}, \beta_{2}$ defined by eq. (4.9) were determined in section 4.3 .

Let us now consider a particular series solution of the full non-homogeneous equation

$$
H^{(n-h)}(\mu, w)=\sum_{n=0}^{\infty} c_{n}^{n-h} w^{n}
$$

the coefficients $c_{n}^{n-h}$ are derived in appendix $\square$ and have the form

$$
\begin{aligned}
c_{n}^{(n-h)}(\mu) & =(-)^{n} \frac{\widetilde{F}_{n}(\mu)}{\prod_{j=1}^{n} f_{0}(j)}=(-)^{n} \frac{\widetilde{F}_{n}(\mu)}{\prod_{j=1}^{n} j^{2}} \\
\widetilde{F}_{n}(\mu) & =\operatorname{det} \widetilde{\mathbb{F}}_{n}(\mu)
\end{aligned}
$$


where $\widetilde{\mathbb{F}}_{n}(\mu)$ is an $n \times n$ matrix similar in form to $\mathbb{F}_{n}(\mu, \sigma)$ except for the last column which depends on the source term expansion:

$$
\begin{aligned}
{\left[\mathbb{F}_{n}(\mu, \sigma)\right]_{i i}=} & k-\sigma-n+i ; \quad\left[\mathbb{F}_{n}(\mu, \sigma)\right]_{i j>i}=-(k+\sigma+n-j) \\
{\left[\mathbb{F}_{n}(\mu, \sigma)\right]_{i+1 i}=} & (\sigma+n-i)^{2} ; \\
& {\left[\mathbb{F}_{n}(\mu, \sigma)\right]_{i n}=-h(\mu) s_{n-i} ; \quad i=1, \ldots, n ; j=1, \ldots, n-1 }
\end{aligned}
$$

where the coefficients $s_{i}$ are defined by the power series expansion of the source term on the right hand side of eq. (4.33):

$$
\begin{aligned}
\frac{h(\mu) w}{(1-w)(1-2 w)^{3}} & =h(\mu) \sum_{n=0}^{\infty} s_{n} w^{n+1} \\
s_{n} & =\sum_{i=0}^{n}(i+1)(i+2) 2^{i-1}
\end{aligned}
$$

it can be checked that both the series expansions for the source term and for $H^{(n-h)}(\mu, w)$ converge for $|w|<1 / 2$, i.e. for $r^{2}-a^{2}<a^{2}$.

Below we write the expansions of $H^{(R)}(\mu, w), H^{(I)}(\mu, w)$ and $H^{(n-h)}(\mu, w)$ up to order $w^{3}$ :

$$
\begin{aligned}
H^{(R)}(\mu, w)= & c_{0}^{R}(\mu)\left(1-k w+\frac{k^{2}}{4} w^{2}+O\left(w^{3}\right)\right) \\
H^{(I)}(\mu, w)= & c_{0}^{I}(\mu)(\log (w)+(-k \log (w)+1+2 k) w \\
& \left.+\frac{1}{4}\left(k^{2} \log (w)+2-3 k^{2}\right) w^{2}+\ldots\right) \\
H^{(n-h)}(\mu, w)= & h(\mu)\left(w+\frac{1}{4}(8-k) w^{2}+O\left(w^{3}\right)\right)
\end{aligned}
$$

In some particular limits the above series can be summed.

First of all let us consider the solution at $\rho \gg a$ to which only the Fourier transform $\widetilde{H}$ at $\mu=0(k=0)$ contributes. In this case, the series in eqs. (4.42) are easily summed and we get:

$$
\begin{aligned}
H^{(R)}(\mu, w) & =1 \\
H^{(I)}(\mu, w) & =\log (w)+\sum_{n=1}^{\infty} \frac{w^{n}}{n}=\log (-w)-\log (1-w)+\text { const. } \\
& =\log \left(\frac{r^{2}-a^{2}}{r^{2}+a^{2}}\right)+\text { const. } \\
H^{(n-h)}(\mu, w) & =\frac{h(\mu)}{2(1-2 w)}-\frac{h(\mu)}{2}=\frac{a^{2} h(\mu)}{2 r^{2}}+\text { const. }
\end{aligned}
$$

Eq.s (4.43) and (4.44) are a very nice confirmation of what we had already established in previous sections considering the limiting form of the equation either for $\mu \rightarrow 0$ or for $a \rightarrow 0$. In particular eq.s (4.43) and (4.44) justify the absolute normalization of the solutions $u_{1,2}$ chosen in eq. (4.13).

Another accessible limit is that corresponding to $\mu^{2} \gg 1 / a^{2}$ with $|w| \ll 1$ (i.e. $r^{2}-a^{2} \ll a^{2}$ ) and keeping $|k w|<1$. This is the asymptotic near-cycle limit in which we approach the brane 
also along $\mathbb{R}^{2}$. We can sum the series up to $O\left(1 / k^{2}\right)$ for the solutions of the homogeneous equation and up to $O\left(1 / k^{3}\right)$ for the particular solution of the non-homogeneous one:

$$
\begin{aligned}
H^{(R)}(\mu, w) & =H_{0}^{(R)}(k w)+\frac{1}{k} H_{1}^{(R)}(k w)+O\left(\frac{1}{k^{2}}\right) \\
H^{(I)}(\mu, w) & =H_{0}^{(I)}(k w)+\frac{1}{k} H_{1}^{(I)}(k w)+O\left(\frac{1}{k^{2}}\right) \\
H^{(n-h)}(\mu, w) & =\frac{1}{k} H_{0}^{(n-h)}(k w)+\frac{1}{k^{2}} H_{1}^{(n-h)}(k w)+O\left(\frac{1}{k^{3}}\right)
\end{aligned}
$$

The functions $H_{l}(k w)$ in the above expressions are obtained by summing the terms proportional to $k^{n-l} w^{n}$ for the solutions of the homogeneous equation and the terms proportional to $k^{n-l-1} w^{n}$ in the series for $H^{(n-h)}(\mu, w)$. This requires the computation as a function of $\mathrm{n}$ of the coefficient of the $k^{n-l}$ or $k^{n-l-1}$ order term in the Hessenberg determinants $F_{n}(\mu, 0)$ and $\widetilde{F}_{n}(\mu)$. Such a computation can be done but, for simplicity, we focus only on the cases $\left.l=0,1\right)$. It can be easily checked 8 that for $l=0$ we have:

$$
\begin{aligned}
H_{0}^{(R)}(\xi) & =c_{0}^{R} \sum_{n=0}^{\infty} \frac{\xi^{n}}{n !^{2}}=c_{0}^{R} I_{0}(2 \sqrt{\xi}) \\
H_{0}^{(I)}(\xi) & =c_{0}^{I} K_{0}(2 \sqrt{\xi}) \\
H_{0}^{(n-h)}(\xi) & =-h(\mu) I_{0}(2 \sqrt{\xi})+h(\mu)
\end{aligned}
$$

where we have set

$$
\xi=|k w|=\mu^{2}\left(r^{2}-a^{2}\right) / 8<1
$$

Once again, eq.s (4.47) are a nice confirmation of what we had predicted in the previous section by the analysis of the differential equation in the near cycle limit. ¿From our general power series solutions we retrieve the result that the regular and irregular solutions of the homogeneous equation in eqs. (4.47) have the same form as the massive Green functions on $\mathbb{R}^{2}$. For a detailed comparison with our previous results it suffices to observe that:

$$
\mu v=\mu \frac{\sqrt{r^{4}-a^{4}}}{2 r} \simeq_{r \rightarrow a} \mu \frac{\sqrt{r^{2}-a^{2}}}{\sqrt{2}}=2 \sqrt{\xi}
$$

Eq.s (4.47) allow also to identify the correct boundary values of the constants appearing in the homogeneous solution. As announced we must have:

$$
c_{0}^{I}=\frac{Q}{4 \pi a^{2}} \quad ; \quad c_{0}^{R}=0
$$

Indeed eq.s (4.47) show that near the cycle the particular integral $H^{(n-h)}$ goes to zero as foreseen in our general discussion.

At first order in $1 / k$ a particular integral of the non-homogeneous equation, is simply $\widetilde{H}^{(n-h)}(\mu, w) \sim h(\mu) / k$ and therefore the behavior of its Fourier transform $H^{(n-h)}(\rho, r)$ in the near-cycle limit is :

$$
H^{(n-h)}(\rho, r) \sim-\frac{1}{2 \pi^{2} a^{4}} \log \left(\frac{\rho}{\rho_{0}}\right)^{2}
$$

${ }^{8}$ For simplicity we assume that the coefficients $c_{0}^{R}$ and $c_{0}^{I}$ do not depend on $\mu$ 
where according to the discussion in section 3 we have assumed the holomorphic function $\gamma$ to be a logarithm (see also the discussion in appendix $\mathrm{C}$ ), and $\rho_{0}$ is an integration constant acting as a regulator, not to be confused with the enhançon radius. The next order terms $(l=1)$ in eq.s (4.46) are written in appendix $\mathrm{G}$.

As far as the asymptotic behavior of the solution at infinity $r \gg a$ is concerned we observe that the first term in the r.h.s. of the equation for $H(\rho, r)$, eq.(4.4), is just a resolved delta function for radially dependent test functions $\Phi(r)$ in the $\mathrm{ALE}_{\mathrm{EH}}$ space:

$$
\lim _{a \rightarrow 0} \int_{A L E}\left[\frac{4}{\pi^{2}} \frac{a^{4}}{r^{8}}\right] \Phi(r) d \Omega_{A L E}=\Phi(0)
$$

Therefore for $r \gg a$ the differential equation (4.4) becomes homogeneous, namely its r.h.s. vanishes. The corresponding equation in the Fourier transform $\widetilde{H}(\mu, r)$, for $w \rightarrow-\infty$ has two independent solutions of which only one is consistent with the boundary condition at infinity (4.33), namely:

$$
\widetilde{H}(\mu, r)=c \frac{\mu}{r} K_{1}(\mu r) \simeq c \times u_{1}(a, \mu, r)
$$

where we have used the radial variable $r$ instead of $w \sim-r^{2} /\left(2 a^{2}\right)$. The coefficient $c$ has already been fixed in terms of the 3-brane charge and the absence of the near cycle regular solution, that is instead divergent at infinity is confirmed. As we expect in this limit, the behavior of the warp factor $H$ is just the same as in the orbifold case [7], schematically:

$$
(\rho, r \gg a): \quad H(\rho, r) \sim \frac{Q}{\left(\rho^{2}+r^{2}\right)^{2}}(1+\log \text { terms })
$$

On the other hand, in the region near the cycle, combining the asymptotic behaviors of the homogenoeus and inhomogeneous solutions, we find

$$
\left(\begin{array}{l}
\rho<a \\
r \sim a
\end{array}\right): \quad H(\rho, r) \sim \frac{Q}{2 a^{2}} \frac{1}{\rho^{2}+v^{2}}-\frac{1}{2 \pi^{2} a^{4}} K \log \left(\frac{\rho}{\rho_{0}}\right)^{2}
$$

where $K$ is a (undetermined at the level of the present analysis) constant. The corrections to the above behavior can be systematically deduced from the power series expansion of the solution described in the present section.

Looking at eq.45.5), it is clear that there could be a value of $r, \rho$ for which $H=0$, this being an indication for the presence of a naked singularity of the repulson type [21]. This singularity should be removed, somehow. In most non-confomal $\mathcal{N}=2$ versions of the gauge/gravity correspondence this singularity has been shown to be excised by the so-called enhançon mechanism [23]. This is the case, for instance, of fractional branes on orbifolds, [0], [16]. The value of the enhançon corresponds to $\rho=\rho_{e}$, the scale where the scalar field $\gamma$ vanishes. This in general turns out to be the scale where the dual gauge theory becomes strongly coupled and new light degrees of freedom are expected to become relevant, both at the gauge theory (where instanton effects become important) and at the supergravity level (where tensionless strings occur). For all this analysis to work, it is important that $\rho_{e}$ is bigger than the scale at which the repulson occurs. In fact, when this is the case, the region where supergravity is reliable, namely $\rho>\rho_{e}$, is free of any singularity. In order to see if this happens also in our case and if the cut-off $\rho_{e}$ has in fact the expected meaning, one should have a full control on the world-volume action of the 
source, with all numerical coefficient, normalization, etc... This we cannot do at the level of the present pure supergravity analysis.

Our solution, however, differs from that of fractional branes on singular space because of a better behaviour of the warp factor $H$ on the plane $\mathbb{R}^{2}$ where it is non-singular, while the solution of the field $\gamma$, which is responsible for the enhançon mechanism, has essentially the same structure. It is then natural to believe that the nice properties described above should not drastically change. Hence we consider our solution to be reliable, well-defined and singularity free for $\rho>\rho_{e}$ (where the leading order near cycle behaviour is well described by the first term in eq. (4.55) itself) while we postpone a more precise analysis of this issue to future work.

\section{Discussion and Conclusions}

In this paper we have constructed a new class of solutions of type IIB supergravity describing 3 -branes whose transverse geometry is of the type $M_{6}=\mathbb{R}^{2} \times A L E$ for an arbitrary smooth $A L E$ manifold. We have shown that these solutions preserve 8 supercharges and thus correspond to $\mathcal{N}=2$ supersymmetry in four dimensions. They are characterized by constant dilaton and axion field, a 3-brane metric

$$
d s^{2}=H^{-\frac{1}{2}}\left(-\eta_{\mu \nu} d x^{\mu} d x^{\nu}\right)+H^{\frac{1}{2}} d z d \bar{z}+H^{\frac{1}{2}} d s_{A L E}^{2},
$$

a self dual five-form

$$
F_{[5]}^{R R}=d\left(H^{-1} \Omega_{\mathbb{R}^{1,3}}\right)+\star d\left(H^{-1} \Omega_{\mathbb{R}^{1,3}}\right),
$$

and a complex three-form, valued only in the transverse space, whose form is uniquely fixed by supersymmetry:

$$
\mathcal{H}_{+}=2 d \gamma_{I}(z) \wedge \omega^{I}
$$

$\left(\gamma_{I}(z)\right.$ being analytic functions on $\mathbb{R}^{2}$ and $\omega_{I}$ harmonic square-integrable anti-self-dual forms on $A L E)$. The whole set of type IIB equations of motion reduces to solving the following PDE for the warp factor $H$

$$
\left(\square_{\mathbb{R}^{2}}+\square_{A L E}\right) H=-\partial_{z} \gamma_{I} \partial_{\bar{z}} \bar{\gamma}_{J} \Delta^{I J}(y)
$$

This equation is quite more involved compared with the analogous equations for the warp factor arising from $\mathcal{N}=1$ supersymmetry which are of the ODE type due to the conical nature of the transverse space. We have then proceeded in studying eq. (5.4) in the simplest instance of an ALE manifold, the Eguchi-Hanson space, whose properties were reviewed in section 1 and appendix B. In the case in which the (here unique) analytic function $\gamma(z)$ possesses radial symmetry, the equation for the warp factor took the form

$$
\frac{1}{\rho} \partial_{\rho} \rho \partial_{\rho} H+\frac{1}{r^{3}} \partial_{r}\left(r^{3} g(r)^{2} \partial_{r} H\right)=-\frac{2 a^{4}}{\pi^{2} r^{8}}|\partial \gamma(z)|^{2},
$$

and, after transforming to the variable $\mu$ conjugate to $\rho$, reduces to a particular type of confluent Heun equation, whose solution by power series can be determined with arbitrary accuracy. Particularly interesting is the behavior of $H(\rho, r)$ near the cycle $r=a$. It is still necessary to invoke an enhançon-like mechanism [23] to cut-off the region $\rho<\rho_{e}$ where supergravity is not reliable. However, contrary to the orbifold case, the warp factor $H(\rho, r)$ is regular as $r \rightarrow a$ for $\rho>\rho_{e}$, due to the fact that the contribution of the three-form to the source term has been regularized. In other words, wherever supergravity is applicable, smoothing out the transverse 
geometry does regularize the solution for the warp factor. The above result can be understood by studying the limit of eq. (5.5) in the presence of an auxiliary source term

$$
\text { source } \propto-Q \frac{\delta(r-a)}{r^{3}} \delta^{2}(z)
$$

on the r.h.s. introduced to fix the boundary conditions near the cycle. In the region $r \rightarrow \infty$ the Laplacian reduces to the flat Laplacian on $\mathbb{R}^{6}$ and $Q$ is the total charge observed at infinity (provided the complex 3-from is vanishing). In the opposite limit, $r \rightarrow a$, the Laplacian reduces to the flat Laplacian on $\mathbb{R}^{4} \times S^{2}$ (the $S^{2}$ dependence of $H$ is trivial) and the sources are clearly regular as long as $\rho \neq 0$, thus so is the solution. By computing the density of charge $q$ on the two-cycle one finds that it is consistent with the interpretation that the three-brane charge is uniformly smeared over the cycle ( $q=Q / \pi a^{2}$, being $\pi a^{2}$ the area of the cycle), thus each element $d \Sigma$ of the surface area of the cycle contributes $q d \Sigma$ to the charge at infinity. The simple form of the Laplacian near the two-cycle, and the fact that we have control over the boundary conditions, allows us to write down the asymptotic form of the metric in that region. Neglecting the contribution of the three-form compared with the source term for the five-form I we find

$$
\left(\frac{1}{\rho} \partial_{\rho} \rho \partial_{\rho}+\frac{1}{v} \partial_{v} v \partial_{v}\right) H \propto-q \delta\left(v^{2}\right) \delta\left(\rho^{2}\right)
$$

which is nothing but the Laplace equation for a point charge in four dimensions written in "bipolar" coordinates. Introducing the variable $R=\sqrt{\left(\rho^{2}+v^{2}\right)}$, denoting the radial coordinate of $\mathbb{R}^{4}$ in units of $q$ we find that $H \sim q / R^{2}$, yielding

$$
d s^{2} \sim \frac{R}{\sqrt{q}}\left(-\eta_{\mu \nu} d x^{\mu} d x^{\nu}\right)+\frac{\sqrt{q}}{R} d R^{2}+\sqrt{q} R d s_{\mathbf{S}^{3}}^{2}+\frac{\sqrt{q}}{R} \frac{a^{2}}{4} d s_{\mathbf{S}^{2}}^{2}
$$

where $d s_{\mathbf{S}^{2}}^{2}$ is the metric of the two cycle of Eguchi-Hanson and $d s_{\mathbf{S}^{3}}^{2}$ is the metric of the threesphere at fixed $R$ in $\mathbb{R}^{4}$. An interesting point is that the above result holds even in the absence of the three-form flux and shows that conformal invariance of the dual theory is always broken because the metric in $R$ and $x^{\mu}$ is no longer of anti de Sitter type. Obviously we cannot take the limit $R \rightarrow 0$, because this would imply going to large curvatures, and in particular crossing the enhançon radius. However, the result suggests that a possible interpretation for the parameter $a$ in the Eguchi-Hanson metric is that of a Fayet Iliopoulos term, breaking conformal invariance in the infrared, in accord with previous work on the subject.

The situation can be summarized as follows. In the absence of flux the exact supersymmetric 3-brane solution that we have found interpolates between a standard ten dimensional 3-brane solution at the singularity of the metric cone on $S^{5}$, i.e. the standard $\mathbb{R}^{6}$ manifold, and a 3brane solution of an effective 8-dimensional supergravity. As explained in the literature 38] (for a review see [39]) we can always consider sphere reductions of all supergravity theories and in particular an $S^{2}$-reduction of type IIB supergravity. This yields an effective 8-dimensional supergravity that has 3-brane solutions. In this case however, there is a coupling to an effective dilaton that emerges as the conformal factor of the metric in the dimensional reduction. Hence the 3-brane solution of the 8-dimensional supergravity is no longer conformal and we can follow the prescription of Townsend et al 40] by making the transition to a dual frame where the metric

\footnotetext{
${ }^{9}$ The argument below becomes exact in the limit where the three-form charge vanishes and it is applicable as long as the $\rho_{e}$ cut-off is not too small.
} 
factorizes into the product of a 3-sphere metric times a domain wall solution of an effective fivedimensional supergravity theory.

Although the main thrust of this paper has been on the supergravity solution, there are various properties of the dual gauge theory that can be described to complete the analysis.

We have seen that supergravity alone is not sufficient to determine $\gamma(z)$ or the boundary conditions for the warp factor, while this is easy in the case of the orbifold (by using for example boundary state techniques) or on the conifold (in which one knows a priori the structure of the source term, despite one does not know the world-volume action). This arbitrariness implies that our solution describes various deformation or various vacua of $\mathcal{N}=2$ theories.

Let us start by taking the orbifold limit and $\gamma(z)=$ const. What we obtain is clearly $(\mathrm{N})$ regular D3-branes at the orbifold point. The constituent branes could move away from the orbifold point in all six transverse directions, thus the dual gauge theory has the relevant hypermultiplets and it is the well known conformal version of an $\mathcal{N}=2$ Yang Mills with product gauge group $\prod_{I=1}^{k} \mathrm{U}\left(\mathrm{d}_{\mathrm{I}} \mathrm{N}\right)$ ( $k$ being the number of irrepses of the discrete kleinian group $\Gamma \subset$ $\mathrm{SU}(2)$ that characterizes the orbifold and $\mathrm{d}_{\mathrm{I}}$ their dimension) and hyper-multiplets in the bifundamental representation. The blow up constitutes no problem in this case, and it is well understood in terms of Fayet-Iliopoulos terms that break conformal invariance but preserve supersymmetry. In the simplest case, namely for $\Gamma=\mathbb{Z}_{2}$, which is the one we focused on, there are three such terms.

On the other hand, in the orbifold limit $(a \rightarrow 0)$, it is known that we may switch on a three form flux described by $\gamma(z) \neq$ const. which preserves the $\mathcal{N}=2$ supersymmetry. In the unbroken phase symmetry requires that we can only have $\gamma(z)=z^{\ell}(\ell>0)$ or $\gamma(z)=\log (z)$ $(\ell=0)$. In the case of a small flux, the corresponding operator on the boundary CFT side can be seen as a perturbation with conformal weight $\Delta=\ell+4$. This perturbation is irrelevant for $\ell>0$, not affecting therefore the conformal IR fixed point, and it is marginal for $\ell=0$, signaling the presence of a small number of fractional branes together with the regular ones at the orbifold point.

The original and perhaps surprising feature of our solution consists in the fact that it may be thought of as the simultaneous effect on the CFT describing regular D3-branes at the orbifold point of the two kind of deformations discussed above: the relevant one related to the blow-up moduli and the marginal/irrelevant one related to the non-trivial flux. Indeed, as previously pointed out, the solution $\gamma(z) \sim \log (z)$ occurs in the orbifold case for the fractional D3-brane solution where the need for a logarithm is dictated by the source term coming from the world volume action [7]. Yet we know that in that case the orbifold cannot be blown up [24] and this can also be understood from the fact that the gauge theory dual to the fractional brane lacks the fields necessary to construct the appropriate Fayet-Iliopoulos terms. One might have deemed that this fact would rule out solutions of this kind in type IIB supergravity: on the contrary, in this paper we have shown that such solutions are indeed possible, although they might not be interpreted as the resolution of fractional branes on orbifolds.

For small fluxes the most obvious interpretation is that we are perturbing the (already resolved) theory on the regular brane as discussed above, but there is also the possibility that some backgrounds with logarithmic behavior correspond to blowing up a fractional brane not at the orbifold point (background $B_{N S}$ field flux through the cycle equal to $1 / 2$ in string units) but at the singular point (with zero flux), where the no-go theorems [24] do not apply. This raises the interesting issue of whether our solution can, in some sense, be seen as interpolating between the SCFT + FI theory with product gauge group and the pure YM theory typical of a fractional brane. 
This is one instance in which supergravity alone is insufficient in determining which is the dual gauge theory and we need string theory inputs as discussed above. This is not too surprising in the case of $\mathcal{N}=2$ theories since, strictly speaking, supergravity alone is never sufficient to determine a dual theory á la Maldacena, (for instance we cannot take the near horizon limit without invoking the enhançon mechanism).

In the future, we plan to return to these issues by making a careful study of the boundary action and trying to test if the above supergravity picture can be confirmed by a microscopic analysis. It would also be interesting to make connection with very recent work on D-instantons [41] and wrapped fivebranes [42] and 433.

\section{Acknowledgments}

We wish to thank P. Di Vecchia, M. Kruczenski, A. Lerda, R. Russo for discussions and very specially M. Billó who has given essential and enlightening advice throughout the whole development of this research project. G.F. wishes to thank the organizers and the participants of the Superstring Theory Workshop in Heraklion (particularly C. Bachas, G.G. Gibbons, E. Kiritsis, U. Lindström, R. Russo and J. Sonnenschein) for the many discussions, I. Klebanov for email correspondence, D. Martelli, B.E.W. Nilsson and D. Tsimpis for discussions regarding related supergravity solutions and U. Gran for software help. M.B. acknowledges support by INFN.

\section{A Notations and Conventions}

The supersymmetric formulation of type IIB supergravity we rely on is that of Castellani and Pesando [32] that uses the rheonomy approach [33]. Hence, as it is customary in all the rheonomy

constructions, the adopted signature of the space-time metric $d \tau^{2}$ used to set the conventions for the gamma matrices is the mostly minus signature:

$$
\eta_{A B}=\operatorname{diag}\{+, \underbrace{-, \ldots,-}_{9 \text { times }}\}
$$

However, in presenting the solution for the metric we used $d s^{2}=-d \tau^{2}$ to conform with more recent usage.

The index conventions are the following ones:

$$
\begin{aligned}
A, B, \ldots=0, \cdots, 9 \quad \text { Flat indices in } D=10 \\
a, b, \ldots=0, \cdots, 3 \quad \text { Flat indices on } \mathbb{R}^{1,3} \\
\alpha, \beta, \ldots=4,5 \quad \text { Flat indices on } \mathbb{R}^{2} \\
t, s, \ldots=6,7,8,9 \quad \text { Flat indices on ALE } \\
\left(i, j, \ldots=4, \ldots, 9 \quad \text { Flat indices on } \mathbb{R}^{2} \times \mathrm{ALE}\right) \\
M, N, \ldots=\text { Curved indices in } D=10 \\
\mu, \nu, \ldots=\text { Curved indices on } \mathbb{R}^{1,3} \\
z, \bar{z}=\quad \text { Curved indices on } \mathbb{R}^{2}
\end{aligned}
$$




$$
\begin{aligned}
& \tau, \sigma, \ldots=\text { Curved indices on ALE } \\
& I, J, \ldots=1,2, \ldots, k \text { indices labeling ALE harmonic self dual 2-forms }
\end{aligned}
$$

The complete vielbein of 10-dimensional space-time are denoted $V^{A}$, those of the $\mathbb{R}^{2} \times \mathrm{ALE}$ manifold without warping factor are denoted $e^{i}$. The convention for constructing the dual of an $n$-form $\omega$ in 10-dimensions is the following:

$\omega=\omega_{A_{1} \ldots A_{n}} V^{A_{1}} \wedge \ldots \wedge V^{A_{n}} \Leftrightarrow \star \omega=\frac{1}{(10-n) !} \epsilon_{A_{1} \ldots A_{10-n} B_{1} \ldots B_{n}} \omega^{B_{1} \ldots B_{n}} V^{A_{1}} \wedge \ldots \wedge V^{A_{10-n}}$

Note that we also use $n$-form components with strength one: $\omega=\omega_{A_{1} \ldots A_{n}} V^{A_{1}} \wedge \ldots \wedge V^{A_{n}}$ and not with strength $n$ ! as it would be the case if we were to write $\omega=\frac{1}{n !} \omega_{A_{1} \ldots A_{n}} V^{A_{1}} \wedge \ldots \wedge V^{A_{n}}$. When it is more appropriate to use curved rather than flat indices then the convention for Hodge duality is summarized by the formula:

$$
\star\left(d x^{M_{1}} \wedge \ldots d x^{M_{n}}\right)=\frac{\sqrt{-\operatorname{det}(g)}}{(10-n) !} g^{M_{1} N_{1}} \ldots g^{M_{n} N_{n}} \epsilon_{L_{1} \ldots L_{10-n} N_{1} \ldots N_{n}} d x^{L_{1}} \wedge \ldots d x^{L_{10-n}}
$$

Applying the rule (A.4) to the case of the specific 10-dimensional metric of our ansatz (3.2) we obtain the following useful formulae for Hodge duals:

$$
\begin{aligned}
\star\left(d z \wedge \Omega_{\mathbb{R}^{1,3}}\right) & =-\mathrm{i} H^{2} d z \wedge \Omega_{A L E} \\
\star\left(d z \wedge \Omega_{A L E}\right) & =\mathrm{i} H^{-2} d z \wedge \Omega_{\mathbb{R}^{1,3}} \\
\star\left(d y^{\tau} \wedge \Omega_{\mathbb{R}^{1,3}}\right) & =-\frac{\mathrm{i}}{2} H^{2}\left(\hat{\star} d y^{\tau}\right) \wedge d z \wedge d \bar{z}=-H^{2}\left(\hat{\star} d y^{\tau}\right) \wedge d x^{4} \wedge d x^{5} \\
\star\left(d z \wedge \omega^{I}\right) & =-\mathrm{i} H^{-1} d z \wedge \omega^{I} \wedge \Omega_{\mathbb{R}^{1,3}} \\
\Omega_{\mathbb{R}^{1,3}} & =d x^{0} \wedge \ldots d x^{3} \\
\Omega_{A L E} & =\sqrt{\operatorname{det}\left(g^{A L E}\right)} d y^{1} \wedge \ldots d y^{4}
\end{aligned}
$$

where $\hat{\star}$ denotes the Hodge dual in the ALE space. The Laplacians on $\mathbb{R}^{2}$ and the ALE space are:

$$
\begin{aligned}
\square_{\mathbb{R}^{2}}= & \left(\frac{\partial}{\partial x^{4}}\right)^{2}+\left(\frac{\partial}{\partial x^{5}}\right)^{2} \\
\square_{A L E}(\cdot)= & -d_{(A L E)^{\hat{\star}} d_{(A L E)}(\cdot)=} \\
& \frac{1}{\sqrt{\operatorname{det}\left(g^{A L E}\right)}} \partial_{\tau}\left[\sqrt{\operatorname{det}\left(g^{A L E}\right)}\left(g^{A L E}\right)^{\tau \sigma} \partial_{\sigma}(\cdot)\right]
\end{aligned}
$$

For the gamma matrices our conventions are as follows:

$$
\begin{aligned}
\left\{\Gamma^{A}, \Gamma^{B}\right\} & =\eta^{A B} \\
\Gamma^{A} & =\left\{\begin{array}{l}
\Gamma^{a}=\gamma^{a} \otimes \mathbf{1} \\
\Gamma^{i}=\gamma_{5} \otimes \tau^{i}
\end{array}\right.
\end{aligned}
$$


where:

$$
\begin{array}{rlrl}
\left\{\gamma^{a}, \gamma^{b}\right\} & =2 \eta^{a b} & ; \gamma_{5} & =-\mathrm{i} \gamma^{0} \gamma^{1} \gamma^{2} \gamma^{3} \\
\left\{\tau^{i}, \tau^{j}\right\} & =2 \eta^{i j}=-2 \delta^{i j} ; \tau_{\bullet}=-\tau^{4} \tau^{5} \tau^{6} \tau^{7} \tau^{8} \tau^{9}
\end{array}
$$

are the gamma matrices in Lorentzian four space and on the six dimensional manifold $M_{6}$ respectively. The matrices $\gamma_{5}$ that anticommutes with all $\gamma^{a}$ and $\tau_{\bullet}$ that anticommutes with all $\tau^{i}$ are respectively hermitian and antisymmetric and we have:

$$
\gamma_{5}^{2}=1 \quad ; \quad \tau_{\bullet}^{2}=-\mathbf{1}
$$

Since the signature of $M_{6}$ is completely negative the $\tau^{i}$ matrices that realize the associated Clifford algebra can be taken to be all real and antisymmetric. They can be further decomposed with respect to the submanifolds $\mathbb{R}^{2}$ and ALE as it follows:

$$
\tau^{i}=\left\{\begin{array}{l}
\tau^{\alpha}=\mathrm{i} \sigma^{\alpha} \otimes \mathbf{1} \\
\tau_{u}=\mathrm{i} \sigma^{3} \otimes \bar{\gamma}_{u}
\end{array}\right.
$$

where $\sigma^{\alpha}=\sigma^{1,2}$ and $\sigma^{3}$ are the standard Pauli matrices while:

$$
\left\{\bar{\gamma}_{u}, \bar{\gamma}_{v}\right\}=\delta_{u v}
$$

are $4 \times 4$ hermitian matrices forming an Euclidean realization of the four-dimensional Clifford algebra.

\section{B The Eguchi-Hanson space}

In the present appendix we state our conventions for the description of the Eguchi-Hanson space. The metric is defined in eq. (4.1) of section 4 , where the 1-forms $\sigma^{i}=\left\{\sigma^{x} \sigma^{y} \sigma^{z}\right\}$ are an explicit realization of the Maurer Cartan forms of the SU(2)-Lie algebra:

$$
d \sigma^{i}=\epsilon^{i j k} \sigma^{j} \wedge \sigma^{k}
$$

and their expression is:

$$
\begin{aligned}
\sigma_{x} & =-\frac{1}{2}(\cos \psi d \theta+\sin \theta \sin \psi d \phi) \\
\sigma_{y} & =\frac{1}{2}(\sin \psi d \theta-\sin \theta \cos \psi d \phi) \\
\sigma_{z} & =-\frac{1}{2}(d \psi+\cos \theta d \phi)
\end{aligned}
$$

Let us now consider the properties of the homology 2-cycle which, in this case, is unique. In the coordinates $(\psi, r, \theta, \phi)$ the cycle is spanned by $(\theta, \phi)$ setting $r=a$. At $r=a$ the Eguchi-Hanson metric has a Bolt singularity which is a coordinate singularity given that $\psi$ has period $2 \pi$. The limit $r \rightarrow a$ is therefore regular for the solution, as can be verified by changing coordinates from $\mathrm{r}$ to $u=r^{2} g(r)^{2}$ and taking $u \rightarrow 0$. Restricting the metric to $\theta, \phi$ with $r=a$ yields:

$$
d s_{\Sigma}^{2}=\frac{a^{2}}{4}\left(d \theta^{2}+\sin \theta^{2} d \phi^{2}\right)
$$

that describes the exceptional cycle $\Sigma$ as a sphere of radius $a / 2$. 
Next let us write the explicit form of the harmonic anti self-dual 2-form $\omega$ fulfilling properties (3.19). Since $\sigma_{z}$ is not globally defined on $\Sigma$ the form:

$$
\omega=\frac{a^{2}}{2 \pi} d\left(\frac{\sigma_{z}}{r^{2}}\right)=\frac{a^{2}}{2 \pi r^{3}} d r \wedge d \psi+\frac{a^{2}}{2 \pi r^{3}} \cos \theta d r \wedge d \phi+\frac{a^{2}}{4 \pi r^{2}} \sin \theta d \theta \wedge d \phi
$$

is closed but not exact and constitutes the only normalizable representative of the second co-

homology group of the space. It can be checked that: $\int_{\Sigma} \omega=1, \int_{A L E} \omega \wedge \omega=-1 / 2=-\mathcal{C}^{-1}$ and that $\star \omega=-\omega$. To check the last property we fix our conventions for the Levi-Civita tensor $\epsilon_{\psi r \theta \phi}=1$ and give the following useful formulae:

$$
\begin{aligned}
\star(d \theta \wedge d \phi) & =-\frac{2 \cos \theta}{r \sin \theta} d r \wedge d \phi-\frac{2}{r \sin \theta} d r \wedge d \psi \\
\star(d r \wedge d \psi) & =-\frac{r}{2 \sin \theta}\left(1-\left(\frac{a}{r}\right)^{4} \cos \theta^{2}\right) d \theta \wedge d \phi-\frac{r \cos \theta}{2 \sin \theta} g(r)^{2} d \theta \wedge d \psi \\
\star(d r \wedge d \phi) & =\frac{r}{2 \sin \theta} g(r)^{2} d \theta \wedge d \psi+\frac{r \cos \theta}{2 \sin \theta} g(r)^{2} d \theta \wedge d \phi
\end{aligned}
$$

where we have used the expression for the determinant of the Eguchi-Hanson metric:

$$
\operatorname{det}\left(g^{A L E}\right)=\left(r^{3} \sin \theta / 8\right)^{2}
$$

Finally the expression (4.3) for the function $\Delta(y)$ is easily derived from the following wedge product:

$$
\omega \wedge \omega=-\Delta(y) \Omega_{A L E} \quad ; \quad \Omega_{A L E}=\frac{r^{3}}{8} \sin \theta d \psi \wedge d r \wedge d \theta \wedge d \phi
$$

\section{$\mathrm{C}$ Analysis of the equation for $\mathrm{H}$ in the Eguchi-Hanson space}

The present appendix is to put on a more rigorous mathematical ground the results on the study of the warp factor $H$ discussed in section 1 and to fix the relevant conventions. The general definition of the Fourier transform $\widetilde{H}(\vec{p}, y)$ ( $\vec{p}$ here is the momentum vector along $\mathbb{R}^{2}$ ) is

$$
\begin{aligned}
\widetilde{H}(\vec{p}, y) & =\frac{\mathrm{i}}{2} \int \frac{d z \wedge d \bar{z}}{(2 \pi)^{2}} \exp [\mathrm{i}(p \bar{z}+\bar{p} z) / 2](H(z, \bar{z}, y)-1) \\
& \equiv \widetilde{H}(p, \bar{p}, y)
\end{aligned}
$$

As already noticed in section 4, in eq.(C.1) the two dimensional momentum vector $\vec{p}$ was substituted by a complex number $p$ whose norm we name $\mu$ :

$$
p=p_{4}+\mathrm{i} p_{5} \quad ; \quad \sqrt{|p|^{2}} \equiv \mu
$$

while $\rho$ denotes the radius in $\mathbb{R}^{2}$. In section 4 we dealt with the case in which $|\partial \gamma(z)|^{2}$ depended only on $\rho$. In this situation we could expect the integral $H(z, \bar{z}, y)$ to depend only on $\rho$ and $r$ since the source term and the coefficients of its differential equation would depend only on these radial coordinates. Since $\gamma(z)$ is required to be analytic, this occurs when $\gamma(z)$ is either a logarithm or a simple power of $z$ :

$$
\gamma(z) \sim\left\{\begin{array}{l}
\log (z) \\
\frac{z^{\alpha}}{\alpha}
\end{array}\right.
$$


Defining the Fourier transform of $|\partial \gamma(z)|^{2}$ :

$$
j(p, \bar{p}) \equiv \frac{\mathrm{i}}{2} \int \frac{d z \wedge d \bar{z}}{(2 \pi)^{2}} \exp [\mathrm{i}(p \bar{z}+\bar{p} z) / 2]|\partial \gamma(z)|^{2}
$$

$\zeta$ being the angular variable on $\mathbb{R}^{2}$. In the two special cases (C.3) the Fourier transformed source term $j$ is a function only of $\mu$ since we can write:

$$
j(\mu)=\frac{1}{(2 \pi)^{2}} \int_{0}^{\infty} \rho d \rho \int_{0}^{2 \pi} d \zeta \exp [\mathrm{i} \rho \mu \cos \zeta]|\partial \gamma|^{2}(\rho)=\frac{1}{2 \pi} \int_{0}^{\infty} \rho d \rho J_{0}(\mu \rho)|\partial \gamma|^{2}(\rho)
$$

which implies 10

$$
j(\mu)=\left\{\begin{array}{lll}
\lim _{\alpha \rightarrow 0} & \frac{1}{2 \pi} \int_{0}^{\infty} \rho d \rho J_{0}(\mu \rho) \frac{1}{\rho^{-2 \alpha+2}} \equiv j(\log ) \rightarrow \infty \\
& \frac{1}{2 \pi} \int_{0}^{\infty} \rho d \rho J_{0}(\mu \rho) \frac{1}{\rho^{-2 \alpha+2}}=\left(\frac{4^{\alpha-1} \Gamma(\alpha)}{\pi\left(\mu^{2}\right)^{\alpha} \Gamma(1-\alpha)}\right)
\end{array}\right.
$$

and where we used the following integral representation of the Bessel $J_{0}$ function:

$$
\int_{0}^{2 \pi} d \zeta \exp [\mathrm{i} x \cos \zeta]=2 \pi J_{0}(x)
$$

Under the hypothesis (C.3) we can denote the unknown function $H$ by $H(\rho, r)$ and its Fourier transform $\widetilde{H}(\mu, r)$ will be given by eq. (4.5). In all other cases $\widetilde{H}=\widetilde{H}(p, \bar{p}, r)$.

However, independently from the explicit form of the twisted field $\gamma(z)$, after partial Fourier transform the original equation (4.4) reduces always to:

$$
L_{r}[a, \mu] \widetilde{H}=J(r, p, \bar{p})
$$

which generalizes eq. (4.6) to a more general choice of $\gamma(z)$ and where the differential operator $L_{r}[a, \mu]$ was defined in eq. (4.7). In eq. (C.8) we have defined the complete source function:

$$
J(r, \theta, \mu) \equiv-\frac{2 a^{2}}{\pi^{2} r^{2}} j(p, \bar{p})+S(\mu, r)
$$

The Wronskian for a basis of two independent solutions $u_{1}(a, \mu, r)$ and $u_{2}(a, \mu, r)$ of the homogeneous equation (4.10) associated with eq. (C.8) is:

$$
W\left(u_{1}, u_{2}\right)=\operatorname{det}\left(\begin{array}{cc}
u_{1}(r) & u_{2}(r) \\
u_{1}^{\prime}(r) & u_{2}^{\prime}(r)
\end{array}\right)=\text { const } \times \frac{1}{p(r)}=\text { const } \times \frac{1}{r^{3} g(r)^{2}}
$$

where the overall constant can be adjusted by choosing the overall normalization of the solutions $u_{1}$ and $u_{2}$.

Focusing on the case in which the source term $J$ depends only on $\rho, r$, the non-homogeneous equation (C.8) can be solved, according to eq. (4.11), in terms of the radial Green function $G\left(a, \mu \mid r, r^{\prime}\right)$ defined by eq. (4.12) plus the the boundary conditions to be imposed on the

\footnotetext{
${ }^{10}$ The Fourier-Bessel transform of $|\partial \gamma|^{2}$ in the logarithmic case is a divergent constant, independent from $\mu$. Formally we name it $j(\log )$ to remind ourselves what it is. At the end, one takes the inverse Bessel-Fourier transform and $j(\log )$ is restored to $1 / \rho^{2}$
} 
solutions $\widetilde{H}$. Following the general theory [37 the Green function is constructed in terms of a basis $u_{1}, u_{2}$ of solutions of the homogeneous equation as follows:

$$
G\left(r, r^{\prime}\right)=\left\{\begin{array}{l}
u_{<}=c_{1}\left(r^{\prime}\right) u_{1}(r)+c_{2}\left(r^{\prime}\right) u_{2}(r) \quad \text { for } \quad r<r^{\prime} \\
u_{>}=d_{1}\left(r^{\prime}\right) u_{1}(r)+d_{2}\left(r^{\prime}\right) u_{2}(r) \text { for } \quad r>r^{\prime}
\end{array}\right.
$$

where the coefficients $c_{i}, d_{i}$ become functions of the second variable and are determined by:

1. the continuity of $G$ at $r=r^{\prime}$ :

$$
u_{<}\left(r^{\prime}\right)=u_{>}\left(r^{\prime}\right)
$$

2. the discontinuity of $1 / w(r)$ in the derivative $\partial G$ at $r=r^{\prime}$ :

$$
\lim _{\epsilon \rightarrow 0}\left\{\left.\frac{d}{d r} G\left(r, r^{\prime}\right)\right|_{r=r^{\prime}+\epsilon}-\left.\frac{d}{d r} G\left(r, r^{\prime}\right)\right|_{r=r^{\prime}-\epsilon}\right\}=\frac{1}{r^{3} g(r)^{2}}
$$

3. The symmetry of the real Green function in the exchange of its arguments:

$$
G\left(r, r^{\prime}\right)=G\left(r^{\prime}, r\right)
$$

4. The boundary conditions at the end-points of the interval $[a, \infty]$ that we want to impose on the function $\widetilde{H}(r)$ :

$$
\lim _{r \rightarrow a} \widetilde{H}=h_{a} \quad ; \quad \lim _{r \rightarrow \infty} \widetilde{H}=h_{\infty}
$$

The same conditions have to be imposed on the Green function.

While the first three items in the above list are mathematical requirements, the fourth encodes the definition of the physical system we want to describe. For this reason we have to choose the boundary conditions by careful comparison with the flat case which is our only guide at this stage. Furthermore, since the solutions $u_{1}, u_{2}$ will be shown to have singular behaviors at the end points of the interval, eq. C.15) has to be understood in the sense of asymptotic regimes.

As shown in [37], the first two conditions become a linear system of equations for the $r^{\prime}$ dependent parameters:

$$
c=c_{1}-d_{1} \quad ; \quad d=c_{2}-d_{2}
$$

with the general solution:

$$
c\left(r^{\prime}\right)=\frac{u_{2}\left(r^{\prime}\right)}{\left(r^{\prime}\right)^{3} g\left(r^{\prime}\right) W\left(r^{\prime}\right)} \quad ; \quad d\left(r^{\prime}\right)=-\frac{u_{1}\left(r^{\prime}\right)}{\left(r^{\prime}\right)^{3} g\left(r^{\prime}\right) W\left(r^{\prime}\right)}
$$

Recalling eq. $(\overline{\mathrm{C} .10})$ if we normalize $u_{1}$ and $u_{2}$ in such a way that the constant appearing in the Wronskian is fixed to 2 :

$$
W\left(u_{1}, u_{2}\right)=\frac{2}{r^{3} g(r)^{2}}
$$

then the Green function takes the form [37]:

$$
G\left(r, r^{\prime}\right)=G_{s}\left(r, r^{\prime}\right)+d_{1}\left(r^{\prime}\right) u_{1}(r)+d_{2}\left(r^{\prime}\right) u_{2}(r)
$$

where:

$$
G_{s}\left(r, r^{\prime}\right)=\left\{\begin{array}{ccc}
\frac{1}{2}\left(u_{2}\left(r^{\prime}\right) u_{1}(r)-u_{1}\left(r^{\prime}\right) u_{2}(r)\right) & \text { for } & r<r^{\prime} \\
0 & \text { for } & r>r^{\prime}
\end{array}\right.
$$


To fix the last bit of information, namely $d_{1}$ and $d_{2}$, we have to impose a choice of boundary conditions. The analysis of sections 4.2 and 4.3 allows to determine the required near cycle behavior of the integral $\widetilde{H}$ functions and consequently both the expressions for the source term $S(\rho, r)\left(4.32\right.$ ) and for the two functions $d_{1}$ and $d_{2}$ :

$$
d_{1}\left(r^{\prime}\right)=-\frac{1}{2} u_{2}\left(r^{\prime}\right) \quad ; \quad d_{2}\left(r^{\prime}\right)=0
$$

which determine in turn the form of the Green function, given in eq. 4.31).

In this final part of the appendix we derive some of the main results of the analysis carried out in section 4.4, besides describing some general feature of eq. (4.33).

Equation (4.33) is characterized by two regular singularities of the homogeneous part at the points $w=0,1$. The regular singularity $w=0$ corresponds to the position of the exceptional cycle $r=a$ while the irregular singularity at $w=-\infty$ corresponds to the large distance limit $r \rightarrow \infty$. The homogeneous equation associated with $(4.33)$ can be framed within the class of equations known as confluent Heun differential equations. A confluent Heun equation may be written in its standard form as follows:

$$
\frac{d^{2} f}{d w^{2}}+\left(4 p+\frac{\gamma}{w}+\frac{\delta}{w-1}\right) \frac{d f}{d w}+\frac{(4 p \alpha w-\sigma)}{w(w-1)} f=0
$$

and in general it has two kind of solutions: an angular solution $f^{(a)}(p, \alpha, \gamma, \delta, \sigma ; z)$ and a radial solution $f^{(r)}(p, \alpha, \gamma, \delta, \sigma ; z)$, defined as solutions of suitable boundary value problems on the intervals $] 0,1[$ and $]-\infty, 0[$ respectively. The homogeneous part of eq. (4.33) is obtained from eq. (C.22) for $\gamma=\delta=1, \alpha=k /(2 p)+1, \sigma=k+2 p$ in the limit $p \rightarrow 0$. Although the literature on this confluent limit of the Heun equation is rather limited, for our purposes we could restrict our analysis of the solution of (4.33) to the neighborhood of the two physically relevant singular points, namely $w=0$ and $w=-\infty$ (i.e. to a region close to the exceptional cycle and to radial infinity) and discuss the restrictions imposed on the solution in these two regions by the boundary conditions on the interval ] $-\infty, 0[$. As far as the singularity $w=0$ is concerned, in its neighborhood we can write a formal power series expansion for an integral of the homogeneous equation:

$$
H(\mu, w)=w^{\sigma} \sum_{n=0}^{\infty} c_{n}(\mu, \sigma) w^{n}
$$

the determination of the coefficients $c_{n}(\mu, \sigma)$ through recurrence relations is a standard procedure in dealing with differential equations with regular singularities. Substituting the above series in the homogeneous equation we obtain:

$$
\begin{aligned}
L_{w} H= & \sum_{n=0}^{\infty}\left(c_{n}(\mu, \sigma) f_{0}(\sigma+n)+c_{n-1}(\mu, \sigma) f_{1}(\mu, \sigma+n-1)+\ldots\right. \\
& \left.+c_{0}(\mu, \sigma) f_{n}(\mu, \sigma)\right) w^{n}=0
\end{aligned}
$$

where the coefficients $f_{0}(\sigma+m)$ and $f_{n>0}(\mu, \sigma+m)$ are determined by the following expansion:

$$
(\sigma+m)(\sigma+m-1)+(\sigma+m) P_{1}(w)+P_{2}(\mu, w)=f_{0}(\sigma+m)+\sum_{n=1}^{\infty} f_{n}(\mu, \sigma+m) w^{n}
$$




$$
\begin{aligned}
f_{0}(\sigma+m) & =(\sigma+m)^{2} \\
f_{1}(\mu, \sigma+m) & =k-\sigma-m \\
f_{n>1}(\mu, \sigma+m) & =-(k+\sigma+m)
\end{aligned}
$$

Hence equation (C.24) gives recursion relations for the unknown coefficients $c_{n}(\mu, \sigma)$. The first of them reads $c_{0}(\mu, \sigma) f_{0}(\sigma)=0$ and yields a non trivial solution only if the indicial equation is fulfilled, namely if $f_{0}(\sigma)=\sigma^{2}=0$. The coefficient $c_{0}(\mu, \sigma)$ is thus a free parameter in terms of which all the other coefficients are expressed. The solution to a generic order $n$ of the recursion relations is represented in eq. (4.36) that we repeat here in a more explicit form:

$$
\begin{aligned}
c_{n}(\mu, \sigma) & =(-)^{n} c_{0}(\mu) \frac{F_{n}(\mu, \sigma)}{\prod_{j=1}^{n} f_{0}(\sigma+j)}=(-)^{n} c_{0}(\mu) \frac{F_{n}(\mu, \sigma)}{\prod_{j=1}^{n}(\sigma+j)^{2}} \\
F_{n}(\mu, \sigma) & =\left|\begin{array}{ccccc}
f_{1}(\mu, \sigma+n-1) & f_{2}(\mu, \sigma+n-2) & \ldots & f_{n-1}(\mu, \sigma+1) & f_{n}(\mu, \sigma) \\
f_{0}(\sigma+n-1) & f_{1}(\mu, \sigma+n-2) & \ldots & f_{n-2}(\mu, \sigma+1) & f_{n-1}(\mu, \sigma) \\
0 & f_{0}(\sigma+n-2) & \ldots & f_{n-3}(\mu, \sigma+1) & f_{n-2}(\mu, \sigma) \\
\vdots & \vdots & & \vdots & \vdots \\
0 & 0 & \ldots & f_{0}(\sigma+1) & f_{1}(\mu, \sigma)
\end{array}\right|
\end{aligned}
$$

Since $\sigma=0$ is a double solution of the indicial equation, from standard theory of differential equations it follows that the homogeneous equation will have a regular solution $H^{(R)}(\mu, r)$ represented by a power series expansion around $w=0$ whose coefficients are $c_{n}^{R}(\mu)=c_{n}(\mu, \sigma=$ $0)$, and an irregular one $H^{(I)}(\mu, r)$, both these independent solutions are defined in eq. (4.35).

As far as the particular solution $H^{(n-h)}(\mu, r)$ of the non-homogeneous equation is concerned, we can write a formal power series expansion as in eq. (4.38). Expanding the source term on the right hand side of eq. (4.33) as in eq. (4.41) and substituting both eq.s (4.38) and (4.41) in eq. (4.33) we obtain:

$$
\begin{aligned}
& L_{w} H^{(n-h)}-\frac{h(\mu) w}{(1-w)(1-2 w)^{3}}=c_{0}^{(n-h)}(\mu) f_{0}(0)+ \\
& \sum_{n=1}^{\infty}\left(c_{n}^{(n-h)}(\mu) f_{0}(n)+c_{n-1}^{(n-h)}(\mu) f_{1}(\mu, n-1)+\ldots\right. \\
& \left.+c_{0}^{(n-h)}(\mu) f_{n}(\mu, 0)-h(\mu) s_{n-1}\right) w^{n}=0
\end{aligned}
$$

¿From the above equation we may deduce the recursion relations for $c_{n}^{(n-h)}(\mu)$ which are solved in eq. (4.39). Such a solution is repeated here in more explicit form:

$$
\begin{aligned}
& c_{n}^{(n-h)}(\mu)=(-)^{n} \frac{\widetilde{F}_{n}(\mu)}{\prod_{j=1}^{n} f_{0}(j)}=(-)^{n} \frac{\widetilde{F}_{n}(\mu)}{\prod_{j=1}^{n} j^{2}} \\
& \widetilde{F}_{n}(\mu)=\left|\begin{array}{ccccc}
f_{1}(\mu, n-1) & f_{2}(\mu, n-2) & \ldots & f_{n-1}(\mu, 1) & -h(\mu) s_{n-1} \\
f_{0}(n-1) & f_{1}(\mu, n-2) & \ldots & f_{n-2}(\mu, 1) & -h(\mu) s_{n-2} \\
0 & f_{0}(n-2) & \ldots & f_{n-3}(\mu, 1) & -h(\mu) s_{n-3} \\
\vdots & \vdots & & \vdots & \vdots \\
0 & 0 & \ldots & f_{0}(1) & -h(\mu) s_{0}
\end{array}\right|
\end{aligned}
$$


Finally let us consider the limit $\mu \gg 1 / a,|w| \ll 1$ and $|k w|<1$ in which the above series expansions can be summed order by order in $1 / k$ according to the expansions (4.46). In eq.s (4.47) we gave the sum of the series at the first non trivial order.

For completeness we give below the sums at the next order in $1 / k$ :

$$
\begin{aligned}
H_{1}^{(R)}(\xi)= & c_{0}^{R} \sum_{n=0}^{\infty} \frac{(-2+n)\left(-n+n^{2}\right)}{3} \frac{\xi^{n}}{n !^{2}}= \\
& c_{0}^{R} \frac{\xi^{3 / 2}}{3} I_{3}(2 \sqrt{\xi}) \\
H_{1}^{(I)}(\xi)= & c_{0}^{I}\left[2 I_{0}(2 \sqrt{\xi})(-1+\xi \log (\xi))+\sqrt{\xi}\left(-\left(I_{1}(2 \sqrt{\xi})(6+(2+\xi) \log (\xi))\right)+\right.\right. \\
& \left.\left.\xi\left(2 K_{3}(2 \sqrt{\xi})+I_{3}(2 \sqrt{\xi}) \log (\xi)\right)\right)\right] \\
H_{1}^{(n-h)}(\xi)= & -\sum_{n=0}^{\infty}\left[s_{0} \frac{-3+2 n-3 n^{2}+n^{3}}{3}-s_{1}\right] \frac{\xi^{n}}{n !^{2}}= \\
& -\frac{h(\mu)}{3}\left(24+24 \xi-24 I_{0}(2 \sqrt{\xi})-2 \xi I_{0}(2 \sqrt{\xi})\right. \\
& \left.+2 \sqrt{\xi} I_{1}(2 \sqrt{\xi})+\xi^{\frac{3}{2}} I_{1}(2 \sqrt{\xi})\right)
\end{aligned}
$$

$\xi$ being $|k w|$.

\section{References}

[1] J. Maldacena, The large $N$ limit of superconformal field theories and supergravity, Adv. Theor. Math. Phys. 2 (1998) 231, hep-th/9711200.

[2] I. R. Klebanov and N. A. Nekrasov, Gravity duals of fractional branes and logarithmic RG flow, Nucl. Phys. B574 (2000) 263, hep-th/9911096.

[3] I.R. Klebanov and A.A. Tseytlin, Gravity duals of supersymmetric $S U(N){ }^{*} S U(N+M)$ gauge theories, Nucl. Phys. B578 (2000) 123, hep-th/0002159.

[4] K. Oh and R. Tatar, Renormalization group flows on D3 branes at an orbifolded conifold, JHEP 05 (2000) 030, hep-th/0003183.

[5] I.R. Klebanov and M.J. Strassler, Supergravity and a confining gauge theory: duality cascades and $\chi$ B-resolution of naked singularities, JHEP 08 (2000) 052, hep-th/0007191.

[6] L.A. Pando Zayas and A.A. Tseytlin, 3-branes on resolved conifold, hep-th/0010088.

[7] M. Bertolini, P. Di Vecchia, M. Frau, A. Lerda, R. Marotta and I. Pesando, Fractional D-branes and their gauge duals, JHEP 02 (2001) 014, hep-th/0011077.

[8] J. Polchinski, $N=2$ gauge-gravity duals, hep-th/0011193.

[9] M. Frau, A. Liccardo and R. Musto, The geometry of fractional branes, Nucl. Phys. B602 (2001) 39, hep-th/0012035. 
[10] O. Aharony, A note on the holographic interpretation of string theory backgrounds with varying flux, JHEP 03 (2001) 012, hep-th/0101013.

[11] M. Petrini, R. Russo and A. Zaffaroni, $N=2$ gauge theories and systems with fractional branes, hep-th/0104026.

[12] O. Aharony, A. Fayyazuddin and J. Maldacena, The large $N$ limit of $N=2,1$ field theories from three-branes in F-theory, JHEP 07 (1998) 013, hep-th/9806159.

[13] B. Brinne, A. Fayyazuddin, S. Mukhopadhyay and D. J. Smith, Supergravity M5-branes wrapped on Riemann surfaces and their QFT duals, JHEP 12 (2000) 013, hep-th/0009047.

[14] C. P. Herzog and I. R. Klebanov, Gravity duals of fractional branes in various dimensions, Phys. Rev. D63 (2001) 126005, hep-th/0101020.

[15] M. Billò, L. Gallot and A. Liccardo, Classical geometry and gauge duals for fractional branes on ALE spaces, hep-th/0105258.

[16] D. Anselmi, M. Billo, P. Fre, L. Girardello and A. Zaffaroni, ALE manifolds and conformal field theories, Int. J. Mod. Phys. A9 (1994) 3007, hep-th/9304135.

[17] M. R. Douglas and G. Moore, D-branes, Quivers, and ALE Instantons, hep-th/9603167.

[18] C. V. Johnson and R. C. Myers, Aspects of type IIB theory on ALE spaces, Phys. Rev. D55 (1997) 6382, hep-th/9610140.

[19] M. R. Douglas, Enhanced gauge symmetry in M(atrix) theory, JHEP 07 (1997) 004, hep-th/9612126.

[20] D. Diaconescu, M. R. Douglas and J. Gomis, Fractional branes and wrapped branes, JHEP 02 (1998) 013, hep-th/9712230.

[21] R. Kallosh and A. Linde, Phys. Rev. D52 (1995) 7137, hep-th/9507022.

[22] P. Aspinwall, Enhanced gauge symmetries and K3 surfaces, Phys. Lett. B357 (1995) 329, hep-th/9507012; W. Nahm and K, Wendland, A hiker's guide to K3: Aspects of $N=(4,4)$ superconformal field theory with central charge $c=6$, Commun. Math. Phys. 216 (2001) 85, hep-th/9912067.

[23] C.V. Johnson, A.W. Peet and J. Polchinski, Gauge theory and the excision of repulson singularities, Phys. Rev D61 (2000) 086001, hep-th/9911161; A. Buchel, A.W. Peet and J. Polchinski, Gauge dual and noncommutative extension of an $N=2$ supergravity solution, Phys. Rev D63 (2001) 044009, hep-th/0008076; N. Evans, C.V. Johnson and M. Petrini, The enhançon and $N=2$ gauge theory/gravity $R G$ flows JHEP 10 (2000) 022, hep-th/0008081; C. V. Johnson, R. C. Myers, A. W. Peet and S.F. Ross, The Enhançon and the Consistency of Excision, hep-th/0105159.

[24] K. Dasgupta and S. Mukhi, Brane constructions, fractional branes and anti-de Sitter domain walls, JHEP 07 (1999) 008, hep-th/9904131.

[25] M. Grana and J. Polchinski, Supersymmetric Three-Form Flux Perturbations on AdS $S_{5}$, Phys. Rev. D63 (2001) 026001, hep-th/0009211. 
[26] S. Gubser, Supersymmetry and F-theory realization of the deformed conifold with three-form flux, hep-th/0010010.

[27] M. Cvetic, H. Lu and C.N. Pope, Brane resolution through transgression, Nucl. Phys. B600 (2001) 103, hep-th/0011023.

[28] M. Cvetic, G. W. Gibbons, H. Lu and C. N. Pope, Ricci-flat metrics, harmonic forms and brane resolutions, hep-th/0012011.

[29] A. Kehagias, New type IIB vacua and their F-theory interpretation, Phys. Lett. B435 (1998) 337 , hep-th/9805131.

[30] M. Grana and J. Polchinski, Gauge / gravity duals with holomorphic dilaton, hep-th/0106014.

[31] J. H. Schwarz, Covariant Field Equations Of Chiral N=2 D = 10 Supergravity, Nucl. Phys. B226 (1983) 269.

[32] L. Castellani and I. Pesando, The Complete superspace action of chiral $D=10, N=2$ supergravity, Int. J. Mod. Phys. A8 (1993) 1125.

[33] L. Castellani, R. D'Auria and P. Fré, Supergravity and Superstring theory: a geometric perspective, World Scientific, Singapore (1990).

[34] K. Becker and M. Becker, M-Theory on Eight-Manifolds, Nucl.Phys. B477 155 (1996), hep-th/9605053.

[35] J. Mc Kay in Proc. Symp. Pure Math., Am. Math. Soc. v37 (1980) 183; P.B.Kronheimer, J. Differ. Geometry 29 (1989) 665, J. Differ. Geometry 29 (1989) 685.

[36] T. Eguchi and A. J. Hanson, Asymptotically Flat Selfdual Solutions To Euclidean Gravity, Phys. Lett. B74 (1978) 249.

[37] P. Dennery, A. Krzywicki, Mathematics for Physicists, Harper and Row 1967;

E. L. Ince, Ordinary Differential Equations, Dover 1956.

[38] H. Lü, C.N. Pope, P.K. Townsend Domain Walls form Anti de Sitter space Phys.Lett. B391 (1997) 39, hep-th/9607164.

[39] P. Fre, Gaugings and other supergravity tools of p-brane physics, hep-th/0102114.

[40] H. J. Boonstra, K. Skenderis and P. K. Townsend, The domain wall/QFT correspondence JHEP01 (1999) 003, hep-th/9807137.

[41] F. Fucito, J. F. Morales and A. Tanzini, D-instanton probes of non-conformal geometries, hep-th/0106061.

[42] J. P. Gauntlett, N. Kim, D. Martelli and D. Waldram, Wrapped fivebranes and N=2 super Yang-Mills theory, hep-th/0106117.

[43] F. Bigazzi, A. L. Cotrone, A. Zaffaroni, N=2 Gauge Theories from Wrapped Five-branes hep-th/0106160. 